IEEE Transactions on Transportation Electrification

Special issue on More Electric Aircraft

\title{
Optimal Design and Synthesis of MEA Power System Architectures considering Reliability Specifications
}

\author{
Angel A. Recalde ${ }^{1 * \dagger}$ Serhiy Bozhko * Jason Atkin * \\ * Department of Electrical and Electronic Engineering, University of Nottingham, NG7 2TU, United Kingdom \\ ¥ School of Computer Science, University of Nottingham, NG8 1BB, United Kingdom \\ † Escuela Superior Politecnica del Litoral ESPOL, EC 090112, Ecuador
}

\{angel.recalde, serhiy.bozhko, jason.atkin\}@,nottingham.ac.uk

\begin{abstract}
Aircraft electrification requires novel designs to supply the growing demand for electric power on-board through efficient and reliable production and distribution of electrical energy. Moreover, the aircraft power system will be a key enabler for the integration of future technologies. Pledging to these intentions, we propose a formulation to synthesize a power system architecture that complies with safety specifications following a Platform Based Design methodology that optimizes the main aerospace drivers. Due to the non-linear nature of the design problem, this paper presents reliability based MILP network design formulations for topology synthesis. The novelty of this approach relies in the adoption of network design optimization for MEA power system construction that allows explicit design formulations as MILP problems. This approach will provide an effective way to include safety specifications by introducing reliability and resiliency constraints.
\end{abstract}

\section{Nomenclature}

$\begin{array}{ll}\text { Sets } & \\ \mathcal{g} & =\text { set of available generators } \\ \mathcal{E} & =\text { set of available distribution paths (generator }- \text { load connections) } \\ \mathcal{N} & =\text { set of available distribution components } \\ \mathcal{A} & =\text { set of available connections between distribution components } \\ K & =\text { set of selected distribution paths (generator }- \text { load connections), } K \subseteq \mathcal{E} \\ F & =\text { set of failure scenarios }\end{array}$

${ }^{1}$ Institute for Aerospace Technology, University of Nottingham 


\section{Generator Selection and Generator-Load Pairing}

$\mathcal{G}=(g, \mathcal{E}) \quad=\quad$ graph containing set of generators $g$ and distribution paths $\mathcal{E}$

$w_{s}, \eta_{s}, \beta_{s}, P_{s}^{G}, \hat{P}_{s}^{G}=$ weight, efficiency, loading, power rating, and power supplied for generator $s$

$L_{l}, r_{\text {TARGET }, l}=$ load demand and reliability target for load $l$

$g_{s} \quad=$ Boolean selection for generator $s$

$y_{s l} \quad=$ Boolean selection for distribution path that connects generator $s$ with load $l$

$r_{V S}, r_{s l} \quad=$ reliability of distribution system or path that connects generator $s$ with load $l$

$u_{s h}, z_{s h, b} \quad=$ weight and efficiency linearization Boolean variables

$w_{h}, \eta_{h b}^{\mathrm{LOSS}} \quad=$ weight and efficiency linearization coefficients

\section{Power Distribution Design}

$\mathcal{G}=(\mathcal{N}, \mathcal{A}) \quad=$ graph containing set of components $\mathcal{N}$ and connections $\mathcal{A}$

$k, r_{\text {TARGET, } k}=$ distribution path $(k \in K, k \in \mathcal{E})$, reliability target for distribution path $k$

$P_{i j, k}, P_{i, k} \quad=$ power flow on connection $i, j$ and component $i$ for distribution path $k$

$c_{i j}, m_{i} \quad=$ fixed cost coefficient for connection $i, j$; fixed cost for component $i$

$c_{i j}^{\mathrm{kW}}, m_{i}^{\mathrm{kW}}=$ variable cost coefficient for connection $i, j$; variable cost for component $i$

$x_{i j}, v_{i} \quad=$ Boolean selection for connection $i, j$ and component $i$

$r_{i j}, r_{i} \quad=$ reliability of connection $i, j$, reliability of component $i$

$d_{k}^{L O A D}, d_{k}^{G E N}=$ load demand and power generation of the distribution path $k$

$z_{i j, k}, z_{i, k}=$ Boolean variables to associate connection $i, j$, component $i$ with distribution path $k$

$f, f_{i j}=$ failure case $f \in F$, failure of connection $i, j$

$q \quad=$ disjoint path that belongs to distribution path $k, q \in\{1,2\}$

$u_{i j, k}, u_{i, k}=$ Boolean that enforces disjointess on paths $q$ for $k$ in connection $i, j$, component $i$

$s_{i, k}^{q} \quad=$ probability of successfully reaching component $i$ while on paths $q$ for $k$

$\sigma_{a b, k} \quad=$ Boolean variable to select two disjoint-path reliability for path $k$

$\gamma_{a, b} \quad=$ two-disjoint path reliability coefficients

$\alpha_{a}, \beta_{b} \quad=$ reliability coefficients for each disjoint path 


\section{I.Introduction}

THE aircraft's Electric Power Distribution System (EPDS) is a network that provides electrical power from the onboard sources (engine-driven generators, back-up systems, ground supplies, etc.) to the loads. These loads comprise all of the devices or sub-systems that provide essential functionality for performing a safe flight. It is expected that on the More Electric Aircraft (MEA) an important percentage of the power demand, including propulsion systems [1], will be fed completely by electricity [2], thus increasing the number of electrically powered devices. With the increasing trend to replace hydraulic and pneumatic systems with electromechanical counterparts on-board [3], EPDS is expected to support an extensive number of configurations based on a larger utilization of power electronics [4] for an increasing number of loads with higher power demands. Certainly, the EPDS will play a vital role in the future aircraft performance and safety. Safety being the paramount attribute for airworthiness qualification in the aerospace industry, it is necessary to investigate the reliability of novel EPDS paradigms and topologies. While it is crucial to provide redundant circuits to feed critical loads when failures occur, it is also important to present an efficient and cost-effective solution. Currently, the standard defined by the aircraft manufacturers guides the conceptual design of an EPDS. This practice has led the subsystem and component suppliers to locally design through a separate optimization approach that is not necessarily optimal with respect to the EPDS network level [5]. The aim of this work is to present an optimization-based design framework that has the potential of synthesizing a MEA EPDS architecture such that it complies with a set of safety specifications to supply critical loads under failure conditions (representing a set of requirements from the standard). The performance evaluation of the potential MEA EPDS is reliabilitycentered (using reliability metrics for both components and system) and the selection of the optimal EPDS candidate relies on the reliability's impact on system's weight and cost. Given recent interest in Mixed Integer Linear Programming (MILP) techniques in other microgrids [6] and transportation electrification problems [7], [8] due to their global-optimum convergence and quicker solving times when compared to other solvers handling the hard-tosolve non-linear version of the problem, MILP is adopted in the design framework. The proposed framework will address an investigation of reliability-based design formulations for the synthesis of MEA EPDS architectures considering the main aerospace drivers, i.e. safety, cost, weight, and efficiency. This approach pretends to extent the few contributions available combining a design framework with MILP reliability-centered optimization. Although MILP is not necessarily unusual in the design of electrical systems in other applications, it is particularly new for the MEA application. The rest of the paper is organized as follows. Literature review in MEA EPDS design is presented 
in Section II. Then, Section III will introduce the EPDS design problem whilst depicting the main implications and difficulties. Later, Section IV will propose an optimization based decomposition method to synthesize an EPDS. This is followed by a linear transformation in Section V needed for solving the problem efficiently. In Section VI, the proposed method will be used to synthesize a MEA EPDS that satisfies a set of reliability specifications; these EPDSs will be assessed in terms of the main aerospace drivers. Finally, the paper will come to a conclusion in Section VII.

\section{Literature Review}

Until recently EPDS designs were typically based on a conventional design flow with a mixture of engineering expertise, practical experience, and general logic. Due to the growth in complexity of power requirements on MEA EPDS and its multi-disciplinary interactions with other aircraft's systems, the design process demands new alternatives over the intuitive expertise of designers to make the initial down-selections and define a design space that is tractable [9]. Following this route, more research has been focused on the design framework proposals [10]-[13], architecture investigation and analysis [14]-[16], EPDS performance evaluation with analytical or simulation techniques [14], [17], [18], optimization-based design models [5], [19]-[21], or a combination of approaches including some forms of design methodology, performance evaluation, and optimal selection [15], [16]. However, MEA EPDS design is still at an early stage and few methodologies in the architecture synthesis direction have been explored.

Among the design frameworks that have been applied to the design of new MEA EPDS architectures there are: Cyber-Physical System [10], [22]-[24], Platform Based Design (PBD) [13], [25], Integrated Design by Optimization [20], [26], and the optimization-based Extended Pareto Front Method [5]. Several contributions have been devoted to the study of MEA EPDS as a Cyber-Physical System, whose concept envisions the integration of computation, communication, control, and sensing technologies [22]. With the introduction of new syntaxes [27] and domainspecific language [24] to satisfy the operational requirements of a MEA EPDS [23], the architecture design of a CyberPhysical MEA EPDS is possible having developed proficiency in new methods [11], control techniques [28], toolboxes [24], [29], [30], and their integration. These techniques and tools have introduced a set of systematic steps

to manage complexity and requirements satisfaction. In regards to architecture design, the Cyber-Physical framework shares with other frameworks a consistent use of optimization routines, with special attention to MILP [7], [10]. Given a set of functional and reliability specifications, PBD incorporates optimization including Correct by Construction [31], and Contract based Design [10] methods. The latter synthesizes an EPDS by iteratively solving an algorithm motivated on a MILP modulo theory [32], where the number of constraints increases on every iteration depending on 
the satisfaction of the reliability requirements. On the other hand, based on the impact analysis of aerospace drivers, Correct by Construction divides the design process into a number of abstraction levels (platforms) and refinement steps that are solved sequentially. There are as many refinement steps as number of platforms until the latest abstraction level is close enough to the physical implementation [13]. In the case of MEA, an EPDS platform is a shared set of common design, engineering, and production efforts, as well as major components over a number of distinct models and types of MEA, often from different but related structure (network). In addition, platforms eliminate large loop iterations and restrict the design space via new structures that provide some design potential for lower cost [33]. The number of intermediate platforms is the essence of PBD and facilitate higher-level optimization. Following the spirit of PBD, this paper presents a design framework that relies on a MILP reliability-based network design which:

- Ensures compliance with a set of reliability requirements by introducing reliability performance constraints in the MILP optimization, that is, performance evaluation is included as design conditions.

- Searches over a design space determined by a set of functional (operational) constraints and major components in order to reach a high-level optimum, that is, system's investigation and analysis is performed during the optimization itself.

- Reduces the necessity of integrating several computational toolboxes and other analytical resources to search the design space and produce a MEA EPDS architecture.

The key EPDS components that define a MEA platform include generation system, power distribution system, and electrical loads. Depending on their desired performance under several conditions (e.g. overloads, harmonics content), sizing of the EPDS components requires some iterations within the aerospace system engineering. The method proposed could potentially mitigate the number of these iterations through earlier abstraction of functionalities (via constraint additions) thus leading to an accelerated design by identification of optimal options. Recent reports have investigated the selection of optimal MEA EPDS [16], [34]-[37], while others have highlighted the importance of architecture synthesis through optimization of reliability, weight, and cost [4], [20], [38]-[40]. For the purpose of assessing reliability, it is vital to translate the safety specifications into a precise set of reliability constraints that accomplishes the expected performance. In a previous investigation [41], a reliability approach for the MEA EPDS design was explicitly formulated as an optimization problem with reliability constraints. Essentially, critical loads which are crucial for flight tasks must be powered at all times and must have alternative power supply routes in case of failure events in EPDS components. This feature has been extensively studied in network design [42]-[48]. In order 
to increase reliability, the designer allocates enough spare capacity such that there are sufficient backup routes to deliver power to prevent critical loads from suffering a power interruption. Therefore, network design techniques can then contribute to efficiently modelling and solving an optimization problem for the MEA EPDS synthesis. It is also possible to improve reliability by considering disjoint-paths for power delivery [48], so that supply on critical loads is not interrupted after failure occurs on an active path. An EPDS can also be designed to ensure power availability on critical loads in the event of component failures [45], connection failures [47], or general failure scenarios [46]. In this case, the system is said to be resistant to failure or resilient [47]. Also, when critical loads are still powered and an EPDS is able to perform its function under a failure scenario, the system is said to be survivable [46]. Resiliency will be modelled within the design formulations in order to achieve high power availability.

\section{Aircraft Power System Design Framework}

A MEA EPDS generates and distributes electrical power on-board. The design of such an EPDS has a large number of degrees of freedom. For instance, given a certain MEA application, several design requirements on physical implementation arise: number of power sources, type of power generation, power train configurations, number of different voltage levels, operational values for the different voltage levels, type of distribution (radial, ring, primary and secondary, etc.), number and type of power converters, technology for power conversion, power conversion switching frequencies, and many other factors that are also related to EPDS's performance (e.g. transient dynamics response, harmonic content, etc.). Certainly, the solution to this design problem is far from trivial (design challenge). Even if a design formulation could be produced, its search space will be prohibitively large due to a combinatorial explosion between all of the technical and economic factors influencing the EPDS's construction, which will make finding an optimum extremely hard. Thus, it is necessary to adopt a design framework that allows efficient designspace exploration and an optimum identification and selection.

\section{A. The MEA EPDS Design Challenge}

In the construction of a MEA EPDS, it is necessary to optimize weight (at minimum cost) while maximizing efficiency and reliability, hence, it can be a multi-objective problem. The system's reliability is determined by the availability of electrical power at the load's terminals. This reliability is enhanced by establishing alternative power paths to make the system resilient and supply critical loads at all times (non-critical loads can be shed if required), which introduces a combinatorial process in the problem formulation. Besides, on-board power generation is usually 
sized according to the maximum load demand and resiliency to failure of one power source, but sizing could include temporal overloads, demand profiles (data models), dynamic response and stability, etc., which introduces interaction of other phenomena and disciplines in the problem. Considering that generator's weight and efficiency is only influenced by its size as shown in Fig. 1 [49], these characteristics become conflicting optimization objectives: larger but lighter generators could run inefficiently most of the time if compared to a heavier system of several generators running either at maximum efficiency or turned off.

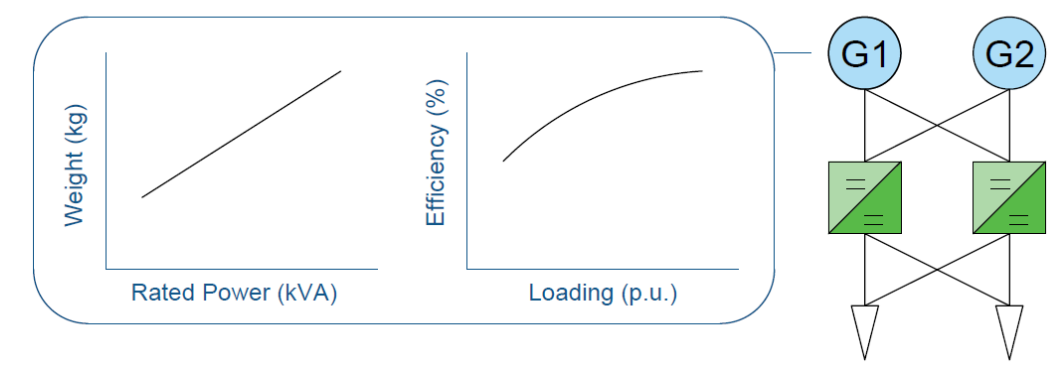

Fig. 1 Aircraft AC synchronous generator's weight and efficiency (left); simple MEA EPDS (right)

Later, the power distribution network should provide several alternate paths to ensure power availability to the loads, which is finally a MEA EPDS resilient architecture. On top of previous complications to formulate the design problem, there is a main difficulty in synthesizing such an EPDS: assessing its reliability is a hard problem to solve. In fact, the EPDS reliability depends on the number of all the possible ways to supply the loads. For example, given the simple EPDS architecture shown in Fig. 1, if both loads were critical, a reliability evaluation technique such as the path-tracing method would deal with a number of $n^{L}=16$ possible paths to supply the loads [ $n$ : ways to supply loads, $L$ : number of loads]. If $n=6$ and $L=8$ (not even a medium sized system yet), an exhaustive evaluation would explore more than 1.5 million combinations! To overcome this, the design framework proposed introduces resiliency characteristics (alternative paths) which prevents combinatorial explosion. To the best of our knowledge, similar approaches have not been applied to the MEA EPDS architecture synthesis application.

\section{B. MEA EPDS Design Framework}

We aim to construct a MEA EPDS that is able to satisfy the demand of a pre-determined group of electricallydriven loads even under component-failure conditions. In general, the EPDS can be built by selecting and connecting a group of power generation and distribution components (topology synthesis). Given a set of power sources (e.g. generators, batteries, energy storage systems, etc.) and distribution components (e.g. rectification or inversion units, 
transformers, cyclo-converters, buses, etc.), the problem is to determine which power sources should supply which loads through which distribution devices, such that all loads are supplied at the reliability level required. Hence, EPDS design requires a selection process to decide which components are required, e.g. by adopting a set of Boolean values ( 0 or 1$)$ to include or not each components/connections in the EPDS. Beyond these selection variables, other decision variables might be used to decide over continuous parameters (sizing), e.g. nominal rating of power sources and converters, power supplied by generators and converters, etc. Commonly, EPDS design requires the definition a priori of certain application-dependent aspects that will limit the search space to some extent. This delimitation will confine components into a library (template or graph) from which a number of components and feasible connections between them will be chosen. This library constitutes the platform of the corresponding abstraction level.

In this paper, the MEA EPDS design space is explored by evaluating the four main aerospace industry drivers (which are also design variables), i.e. $\operatorname{cost} C$, weight $W$, efficiency $\eta$, and reliability $r$. To do so, it is compelling to observe the following relations between $C, W, \eta$ and $r$ in the aircraft industry [31]:

- The number of generators and their power ratings drives the trade-off between cost and efficiency

- The reliability performance of the EPDS is assessed as the availability of power at loads' terminals

- The cost of power distribution is driven by the generator-load combination and the topology of the EPDS

- The weight of the power conversion is driven by a power density $(\mathrm{kW} / \mathrm{kg})$ and the amount of power to convert, thus, it is independent from the generator-load combination

The above points represent the insights to redefine the MEA EPDS design. Given these insights, EPDS generation and distribution is amenable to be designed sequentially by adopting a PBD-inspired methodology. Through a series of refinement steps between abstracted platforms, PBD can implement a MEA EPDS architecture [50]. The proposed framework is depicted in Fig. 2, where the MEA design challenge (left) is undertaken by the design framework proposed (right) with the help of the aircraft industry's insights. The resultant platform is a synthesized MEA EPDS Architecture. 

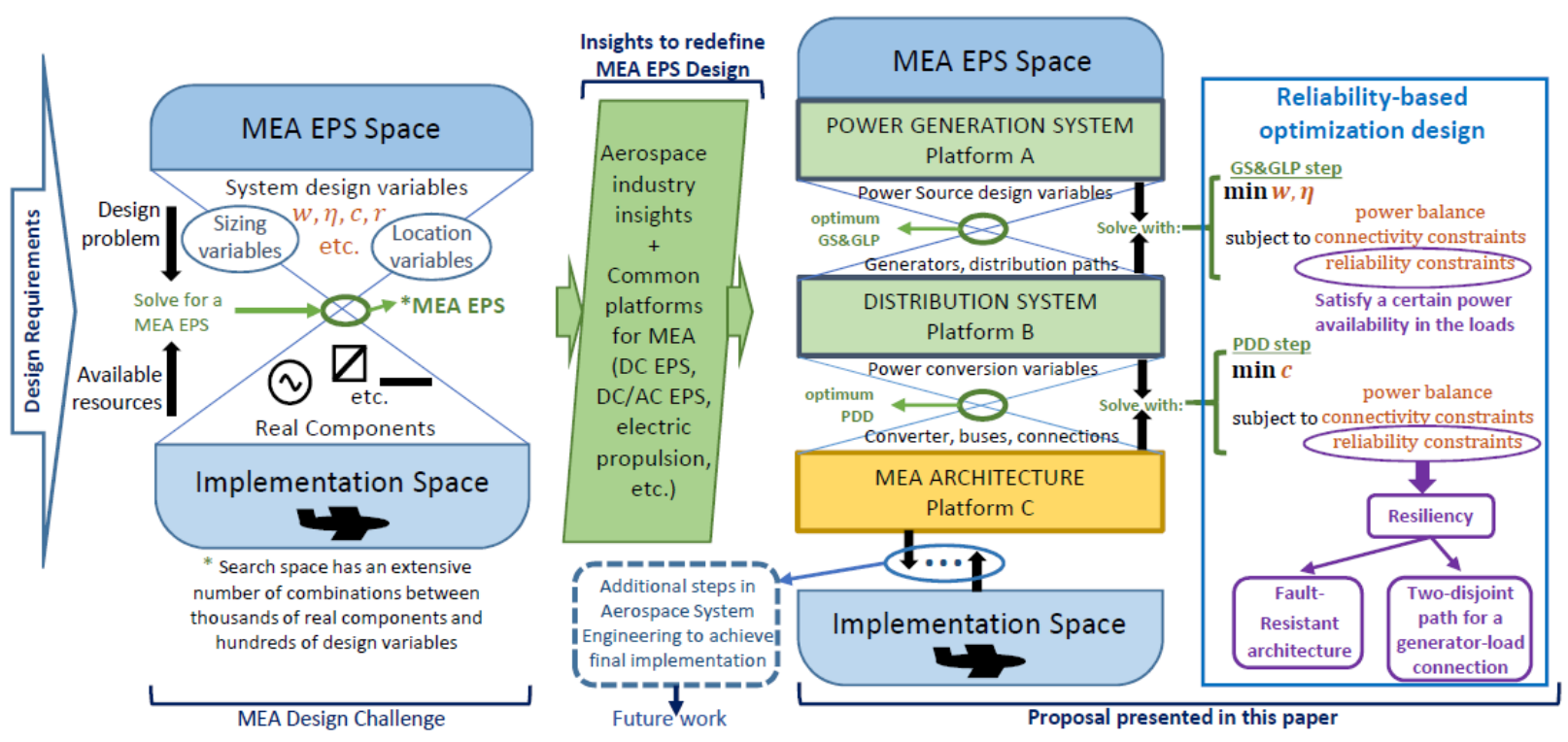

Fig. 2 MEA EPDS Design Framework; design challenge (left), framework proposal (right)

The design exercise starts with a set of design specifications and requirements. After abstracting the intermediate platforms based on the aerospace industry insights and the disruptive platforms envisioned for MEA [2], [4], [16], [51] (see Fig. 2 POWER GENERATION SYSTEM or Platform A, and DISTRIBUTION SYSTEM or Platform B), a MEA EPDS architecture can be synthesized (Platform C). Each refinement step between any two platforms demands a reliabilitybased design optimization to ensure performance compliance at an optimum $C, W, \eta$ and $r$. The POWER GENERATION SYSTEM platform (Platform A) solves for the optimal amount, size, and assignment of generated power to the loads (load allocation). This platform uses some abstraction from the distribution system (Platform B) to provide a generatorload path. Then, the DISTRIBUTION SYSTEM (Platform B) is refined into an optimal EPDS network which finally forms, with the optimal power generation system of Platform A, the MEA ARCHITECTURE or Platform C. This architecture can be consecutively refined until the MEA EPDS can be implemented on a real MEA aircraft. However, the final implementation is out of the scope of this paper. Whenever the design specifications change, it is possible to adapt the proposed framework with new constraints or intermediate abstractions, so that it can fit in the aerospace system design iterations. The refinement between Platform A and B is known as Generation Selection and Generator-Load Pairing (GS\&GLP step), and the refinement between Platform B and C is known as Power Distribution Design (PDD step). The former, explained in Section IV.B, will determine the number of generators, their ratings, and the generator-load pairs arrangement, while the latter, detailed in Section IV.C, builds a EPDS topology with the previous step's solution [31]. 


\section{Formulation for the Design of Aircraft Power System}

The MEA EPDS architecture (Platform C in Fig. 2) consists of a group of interconnected generators and distribution components that supply power to the loads through several paths to provide resiliency. Synthesizing such an EPDS from scratch requires the utilization of decision variables to select and determine a series of parameters to allow generation and distribution. Concerning the selection process, Boolean decision variables ( 1 if selected, 0 otherwise) will determine which components or connections are selected. For example, a binary variable $g_{j}$ ( 0 for no, 1 for yes) can determine whether generator $s$ is selected, the existence of a connection from $i$ to $j$ can be determined by a binary variable $y_{i j}$ (or $y_{j i}$ for the reverse connection, set to 1 if the connection exists or 0 otherwise), and similarly with the rest of the distribution components. On the other hand, continuous decision variables could be used to determine the system's performance, i.e. $\operatorname{cost} C$, weight $W$, efficiency $\eta$, reliability $r$, and other parameters, i.e. power flow $P_{i j}$ over connection $y_{i j}$, etc. In some cases, these performance indicators and parameters are functions of decision variables, e.g. cost $C$ could be function of $g_{j}, y_{i j}, r$, and $P_{i j}$, weight $W$ might be function of $g_{j}, y_{i j}$, and $P_{i j}$, and generation efficiency may be a function of power rating and loading, etc. In most cases, these functions are non-linear (see $\eta$ in Fig. 1) or deserve a time-consuming evaluation (as in the case of system's reliability, see Fig. 1). A general formulation that pursue a design goal may include: (1) optimization of $C, W, \eta$ and $r$, (2) power balance, and (3) achievement of a certain reliability target. This formulation is compactly written below.

$$
\begin{gathered}
\text { minimize } C, W \& \text { maximize } \eta, r \\
\text { subject to } P_{\text {GenERAtors }}=P_{\text {LOAdS }}+P_{\text {LOSSES }} \\
r \geq r_{\text {TARGET }}
\end{gathered}
$$

The optimization goals in (1.1) are conflicting, and the reliability in (1.3) depends on the specific combination of system components chosen and the way in which they are arranged, which is a huge number of combinations. Given the difficulties in solving formulation (1.1)-(1.3), the design framework in Fig. 2 (Section III) is applied.

\section{Generator selection and generator-load pairing (GS\&GLP) step}

The GS\&GLP step attempts to select a number of generators, determine their ratings (sizing), and allocate a set of loads to each generator, i.e. produce a set of generator-load pairs which meet the loads' demand and reliability requirements. An example of such an assignment is shown in Fig. 3. From a library of available generators and feasible connections forming a template in Fig. 3(a) (Platform A in Fig. 2), the GS\&GLP step produces the solution shown in 
Fig. 3(b) (the optimum of the first refinement step); a template is a graph $\mathcal{G}=\{g, \mathcal{E}\}$ where $g$ is the set of generators (1 to 4 in this case), and $\mathcal{E}$ is the set of generator-load connections ( 1 to $\left\{\mathrm{L}_{1}, \mathrm{~L}_{2}\right\}, 2$ to $\left\{\mathrm{L}_{1}, \mathrm{~L}_{3}\right\}$, etc.) which are referred as distribution paths for the rest of the paper. These distribution paths will be later refined in the PDD step.

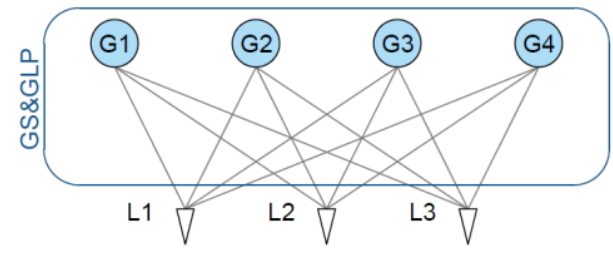

(a)

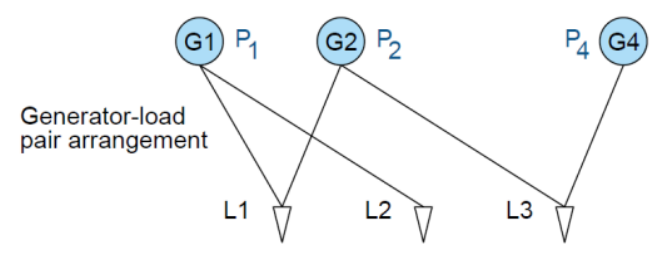

(b)

Fig. 3 GS\&GLP problem (a) template; (b) power ratings and pairing arrangement

The GS\&GLP problem can be formulated as the minimization of all $w_{s}$ (generator $s$ weight) and the maximization of all $\eta_{s}$ (generator $s$ efficiency). It will be assumed that cost is directly proportional to weight, hence weight minimization is equivalent to cost minimization $(C \propto W)$. Let the selection of a generator $s$ be performed using a Boolean $g_{s}$ (1 if $s$ is selected, 0 if not). Also, let this generator have a power rating $P_{s}^{G}$ and be connected to a load $l$ with demand $L_{l}$; the connection between generator $s$ and load $l$ can be represented by Boolean $y_{s l}$ ( 1 if connection exists, 0 if not). The total power supplied by generator $s$ is $\sum_{s} L_{l} y_{s l}$ and its loading factor $\beta_{s}$ is $\sum_{s} L_{l} y_{s l} / P_{s}^{G}$. Recalling that $w_{S}$ can be considered as a function of $P_{S}^{G}$ and nominal speed (RPM), and that $\eta_{s}$ can be expressed as a function of $P_{s}$ and $\beta_{s}$ (see Fig. 1), then the GS\&GLP problem can be written as in (2.1). The product of $w_{s}$ and $\eta_{s}$ by $g_{s}$ in (2.1) allows to set weight and efficiency of generator $s$ to 0 when it is not selected.

$$
\min _{w, \eta, g, y}\left(\sum_{s} w_{s} g_{s}\right), \max _{w, \eta, g, y}\left(\eta_{1} g_{1}\right), \max _{w, \eta, g, y}\left(\eta_{2} g_{2}\right), \quad \ldots \max _{w, \eta, g, y}\left(\eta_{s} g_{s}\right)
$$

The result of (2.1) comprises the selection of generators $g_{s}$, their power ratings $P_{s}^{G}$, and the generator-load pairs $y_{s l}$ (or distribution paths). In specific applications, several technical aspects are decided a priori, e.g. operating speed, type of electrical machines (e.g. PMSM), geared or non-geared powertrain, maximum number of generators, etc. Along with (2.1), a set of connectivity and reliability constraints are included. Connectivity ensures power balance, as in (1.2). The reliability constraints ensure required power availability at load terminals. An EPDS's reliability will depend on the reliabilities of the generators $\left(r_{s}\right)$, and the reliability of the distribution paths. The former are frequently available from manufacturers' datasheets. However, the latter is unknown at this stage. To overcome this uncertainty, the distribution system will be depicted as a virtual system VS (containing the set of $y_{s l}$ distribution paths) whose 
reliability $r_{V S}$ can now be specified in three ways: 1 ) as a lower boundary $\underline{r_{V S}}$ (a minimum reliability will be assumed to be achievable in the PDD step), 2) as a single variable $r_{V S}$, or 3) as a set of variables $r_{s l}$ (one for each $y_{s l}$ ). In any case, for a load connected to a generator $s$, the reliability can be written as in (2.2):

$$
r_{V S} r_{S} \geq r_{\mathrm{TARGET}, l}
$$

The left-hand side of (2.2) is the reliability of a series system assuming independent components' reliabilities. Furthermore, a load which is connected to different generators through independent paths, would be powered unless all generators/paths fail, a probability which can be expressed as in (2.3):

$$
1-\prod_{s}\left(1-r_{V S} r_{S}\right) \geq r_{\text {TARGET, } l}
$$

That is, (2.3) is the reliability of a parallel-series system. The optimal solution for the GS\&GLP comprises:

- A group of selected generators $(\subseteq g)$ with their corresponding ratings $P_{s}^{G}$

- A set $K$ containing the group of selected connections $y_{s l}$ (each $y_{s l}$ supplies load $l$ from generator $s$ ), i.e. $K=$ $\left\{k_{1}, k_{2}, \ldots\right\}$ for which $y_{s l}=1$, where $k$ represents a selected distribution path $y_{s l}$, and $K \subseteq \mathcal{E}$

- The reliability of the distribution system, either as $\underline{r_{V S}}, r_{V S}$, or a set of $r_{s l}$ (one per selected $y_{s l}$ ).

The optimal GS\&GLP solution is used in the PDD step (next refinement step) explained below.

\section{Power Distribution Design (PDD) step}

Considering the synthesized optimal power generation system (Platform A in Fig. 2) from the previous GS\&GLP refinement step, the PDD step attempts to find a topology for the MEA EPDS such that the resultant platform is the MEA EPS architecture (Platform C in Fig. 2), which is the object of investigation in this paper. Hence, PDD selects a group of distribution components and connections to construct or refine the distribution paths of the GS\&GLP step. The PDD problem can also be formulated as (1.1). Following the $C \propto W$ assumption, PDD refinement step can be expressed as a cost minimization problem subject to connectivity and reliability constraints.

Consider a template represented with a graph $\mathcal{G}=\{\mathcal{N}, \mathcal{A}\}$ where $\mathcal{N}$ is a set of distribution devices (converters, buses, etc.), and $\mathcal{A}$ is a set of connections (conductors, contactors, etc.) between components in $\mathcal{N}$, see Fig. 4(a). Let a connection between components $i, j$ exist if a Boolean $x_{i j}$ is set. Also, let a component $i$ be selected if a Boolean $v_{i}$ is set. The distribution system's total cost comprises a fixed cost (e.g. installation cost) and a variable cost that depends

on the amount of power transferred. Let $x_{i j}$ have a fixed $\operatorname{cost} c_{i j}$, a variable $\operatorname{cost} c_{i j}^{k W}$, and a power flow $P_{i j}$ that is the 
summation of all existing power flows $P_{i j, k}$ per distribution path $k$, i.e. $P_{i j}=\sum_{k \in K} P_{i j, k}$. Similarly, let $v_{i}$ have a fixed cost $m_{i}$, a variable cost $m_{i}^{k W}$, and a power flow $P_{i}$ which is the summation of all transferred power flows $P_{i, k}$ per distribution path $k$, i.e. $P_{i}=\sum_{k \in K} P_{i, k}$. The distribution system's fixed cost is the summation of all the connection's $c_{i j} x_{i j}$ and all the component's $m_{i} v_{i}$ fixed costs. The variable cost is proportional to the amount of power transferred through connections $\left(P_{i j}\right)$ and components $\left(P_{i}\right), \sum_{k \in K} P_{i j, k}$ and $\sum_{k \in K} P_{i, k}$ respectively. Hence, the variable cost for connections is $c_{i j}^{\mathrm{kW}}\left(\sum_{k \in K} P_{i j, k}\right)$ and the variable cost for components is $m_{i}^{\mathrm{kW}}\left(\sum_{k \in K} P_{i, k}\right)$. Then, the total cost of the distribution system can be written as in (3.1).

$$
\min _{P, x, v} \sum_{(i, j) \in \mathcal{A}}\left(c_{i j} x_{i j}+c_{i j}^{\mathrm{kW}}\left(\sum_{k \in K} P_{i j, k}\right)\right)+\sum_{i \in \mathcal{N}}\left(m_{i} v_{i}+m_{i}^{\mathrm{kW}}\left(\sum_{k \in K} P_{i, k}\right)\right)
$$

$P_{i, k}$ can be defined as the summation of all the power from incoming (or outgoing) connections, i.e. $P_{i, k}=\sum_{j} P_{j i, k}$, so that all connections and components' power flows can be represented with $P_{i j, k}$ flows. The reader can refer to Fig. 4(b) for the relations between $P_{i j}, P_{i}, P_{i j, k}, P_{i, k}$, and the distribution path $k$. The variable cost can be thought of a proportional cost that depends on the size of the system (which depends on the amount of power transferred). Therefore, connections and components are sized accordingly (e.g. conductor gauge, converter size). Alternatively, $c_{i j}$ can include both component cost and connection cost, thus eliminating the $m_{i} v_{i}$ term in (3.1). This simplification is viable when modularity is required, e.g. addition of customized converters with swappable boards whose number of boards depends on the power requirements, bus-bars systems, etc.

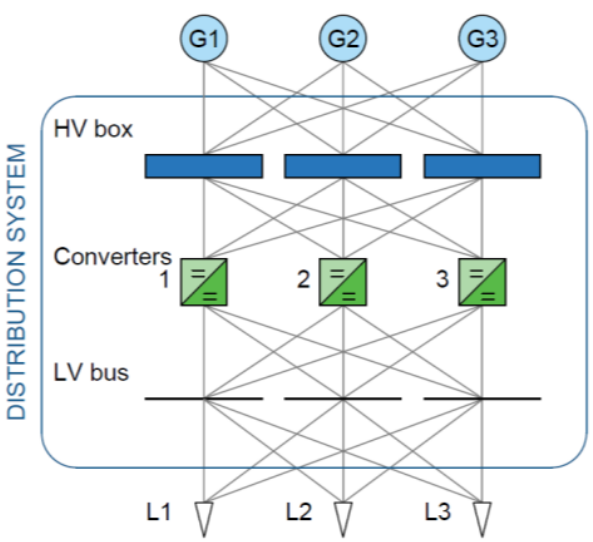

(a)

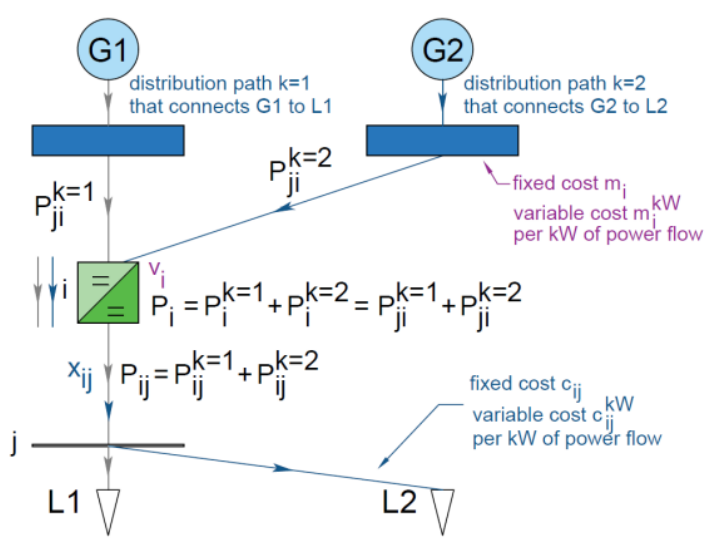

(b)

Fig. 4 PDD problem (a) template; (b) detail example of power flows on a section of the EPDS 
Following (1.1), efficiency might be included in (3.1). The EPDS distribution's efficiency depends mainly on the type of power conversion technology and other implementation factors, e.g. equipment location, load type, etc. In the PDD platform, power conversion functional model relies on the voltage step-up/down for a certain power transmission whose weight depends on a power density figure. Further refinement steps are needed so that the abstraction of efficiency-related functional specifications are possible and power conversion topologies are explored [52], hence, efficiency is deferred for future research works. A group of constraints on connectivity, flow balance (1.2), and reliability are added to (3.1). Assuming independent failure events, with $r_{i j}$ denoting the reliability of the $i, j$ connection and $r_{i}$ the reliability of component $i$, the $k$ distribution path's reliability is the reliability of a series system:

$$
\left(\prod_{j \mid(i, j) \in k} r_{i j}\right)\left(\prod_{i \in k} r_{i}\right) \geq r_{\mathrm{TARGET}, k}
$$

Equation (3.2) contains of all the components and connections that form the path $k$. The reliability target $r_{\text {TARGET,k}}$ of the distribution path $k$ in (3.2) must match the reliability of the distribution system found in the GS\&GLP step, that is, $r_{\text {TARGET }, k}$ (equal to $\underline{r_{V S}}, r_{V S}$, or $r_{S l}$ ). If (3.2) is unable to meet $r_{\text {TARGET, } k}$, an expression to allow alternative paths is required. The addition of alternate paths increases the system's reliability and incorporates resiliency in the EPDS. Considering a number of $w$ alternate independent paths that connect the generator and load of the distribution path $k$, the reliability can be calculated as the left hand side of (3.3), which must exceed some minimum value $r_{\mathrm{TARGET}, k}$.

$$
1-\prod_{w}\left(1-\left(\prod_{j \mid(i, j) \in k} r_{i j}\right)\left(\prod_{i \in k} r_{i}\right)\right) \geq r_{\text {TARGET, } k}
$$

The higher the number of alternative paths, the higher reliability is obtained, at the cost of increasing the size of the system. Hence, there is a compromise between reliability, cost, and weight in the PDD step that leads to a trade-off. The number of alternate routes can be determined either by strengthening the EPDS for a set of failure scenarios, e.g. prepare for all single-component failures, or building disjoint alternative paths [48], [53] for powering critical loads. While the former requires the incorporation of a failure set, the intention of the latter is to increase the probability that at least one path delivers power to critical loads. Thus, the reliability constraints in (3.2)-(3.3) will be reformulated to perform a reliability-based or resilient network design. The resilient network design aims to synthesize a resilient EPDS architecture and could be used for obtaining the trade-off between reliability, cost, and weight. 
Summarizing this section, a PBD-inspired design framework is used to solve (1.1)-(1.3) in two steps: GS\&GLP (2.1)-(2.3) and a PDD (3.1)-(3.3). The formulations given for the GS\&GLP and PDD refinement steps are non-linear and hard to solve. Therefore, in the following section we propose a linear transformation (linearization) to produce a MILP formulation that can be solved in polynomial time (in terms of number of variables and constraints) by a MILP solver, to reach guaranteed optimality.

\section{Linear Transformation of EPDS Design}

In order to avoid the use of heuristic techniques [48], [53], decrease the optimization complexity, and utilize an off-the-shelf MILP-solver, linearization techniques based on discretization, piecewise linear functions, and other equivalent linear models are used to solve the non-linear formulations in the GS\&GLP and PDD refinement steps.

\section{E. Generator selection and generator-load pairing}

The GS\&GLP problem in (2.1) is non-linear and multi-objective: total weight minimization and efficiency maximization for each generator. One alternative to linearize (2.1) consists in reformulating it as a linear combination of objectives. In this case, each objective has an importance determined by coefficients $a_{1}, a_{2}, \ldots, a_{n}$. In addition, efficiency $\eta_{s}$ is expressed in terms of losses $\left(\eta_{s}^{\text {LOSS }}\right)$ to allow the optimizer to minimize total generation losses; the reader is referred to Appendix 1 for obtaining $\eta_{s}^{\text {LOSS }}$ from $\eta_{s}$. Then, (2.1) can be written as:

$$
\min _{w, \eta, g, y} \sum_{s}\left(a_{1}\left(w_{s} g_{s}\right)+a_{2}\left(\eta_{s}^{\text {LOSS }} g_{s}\right)\right)
$$

Equation (4.1) still contains non-linear products of continuous $\left(w_{s}, \eta_{s}^{\mathrm{LOSS}}\right)$ and Boolean $\left(g_{s}\right)$ variables. To deal with this, it is possible to discretize $w_{s}$ and $\eta_{s}^{\text {LOSS }}$ (the optimizer will search over a finite set of values). An advantage of discretizing $w_{s}$ is that commercial values (discrete generator sizes) could be used. In discretization, only one of the possible values must be selected (unique value). Let a Boolean $u_{s h}$ select a unique value for the power rating $P_{h}^{G}$ and weight $w_{h}$ of generator $s$, and let another Boolean $z_{s b}$ select a unique generation loss $\eta_{h b}^{\text {LOSS }}$ and supply power $\hat{P}_{h b}^{G}$ that correspond to the selected $P_{h}^{G}$. In this case, $\sum_{h} u_{s h}=1, \sum_{h} \sum_{b} z_{s h b}=1$ so that generator $s$ has only one value on each of its parameters $P_{h}^{G}, w_{h}, \eta_{h b}^{\text {LOSS }}$ and $\hat{P}_{h b}^{G} . w_{s}$ is now a vector of $h$ different values $w_{h}$, and $\eta_{s}^{\text {LOSS }}$ is a replaced with a vector of $b$ different loadings $\eta_{h b}^{\text {LOSS }}$ corresponding to the rating $h$. Then, (4.1) can be rewritten as:

$$
\min _{u, z, y} \sum_{s}\left(a_{1}\left(\sum_{h} w_{h} u_{s h}\right)+a_{2}\left(\sum_{h} \sum_{b} \eta_{h b}^{\text {LOSS }} z_{s h b}\right)\right)
$$


$w_{s}$ and $\eta_{s}^{\mathrm{LOSS}}$ can also be approximated by piecewise linear functions (PWL). PWL places a linear segment between a pair of discrete values, thus, the optimal solution can take an intermediate values. Solvers could handle convex functions $\left(w_{s}, \eta_{s}^{\mathrm{LOSS}}\right)$ with less work than non-convex functions (convexity is a degree of the function's curvature and discontinuity). Now, connectivity and reliability constraints will be added to (4.2). Each generator must have a unique rating (or power rating $=0$ if generator is not chosen), then

$$
\sum_{h} u_{s h} \leq 1 \quad \forall s
$$

Within rating in (4.3), a unique generation loss value must be selected (or 0 if generator is not chosen):

$$
\sum_{b} z_{s h b} \leq u_{s h} \quad \forall s, \forall h
$$

A load $l$ can be connected to generator $s$ only if that generator is selected (4.5).

$$
y_{s l} \leq \sum_{h} u_{s h} \quad \forall l, \forall s
$$

The total load power cannot exceed the selected generator rating $P_{h}^{G}$ for any generator (4.6); similarly, the total load power cannot exceed the generator's supply power $\hat{P}_{h b}^{G}$ (considering losses) (4.7)

$$
\begin{array}{lll}
\sum_{l} L_{l} y_{s l} & \leq \sum_{h} P_{h}^{G} u_{s h} & \forall s \\
\sum_{l} L_{l} y_{s l} \leq \sum_{h} \sum_{b} \hat{P}_{h b}^{G} z_{s h b} & \forall s
\end{array}
$$

Note that the supply power $\hat{P}_{h b}^{G}$ depends on $\beta_{s}$. Lastly, (4.8) ensures each load is connected at least to one generator.

$$
\sum_{s} y_{s l} \geq 1 \quad \forall l
$$

The reliability constraints in (2.2)-(2.3) are now linearized. By taking the logarithm of the product in (2.2) and (2.3), a summation results. Then, this summation is a linear reliability constraint that can be solved in two ways depending on the value of the distribution system's reliability. Recalling that the distribution system's topology is unknown at this stage, its reliability can be assumed to be $\underline{r_{V S}}$ (achievable lower boundary), or it can be introduced as a variable (single variable $r_{V S}$ for whole distribution system, or set of variables $r_{S l}$ for each distribution path) [8]. For the former, the reliability constraint is written as (4.9).

$$
\sum_{S} y_{S l} \ln \left(1-\underline{r_{V S}} r_{S}\right) \leq \ln \left(1-r_{\mathrm{TARGET}, l}\right) \quad \forall l
$$


where $r_{\text {TARGET, } l}$ is the reliability target for load $l$. Note that each term $\ln \left(1-\underline{r_{V S}} r_{s}\right)$ is constant, therefore (4.9) is linear. For the latter, the reliability of the distribution system $\left(r_{V S}\right.$, or the set $\left.r_{S l}\right)$ replaces $\underline{r_{V S}}$ in (4.9). However, this reliability is now a variable that falls inside the logarithm and requires further linearization (a variable inside a logarithm is a non-linear expression). Similarly to $w_{S}$ and $\eta_{s}^{\text {LOSS }}$, we can discretize $y_{S l} \ln \left(1-r_{V S} r_{s}\right)$ into a set of possible reliability values $r_{S o}^{E}$ (subscript $o$ accounts for the discretized values of $\ln \left(1-r_{V S} r_{S}\right)$ ). Then, (4.10) and (4.11) are the reliability constraints when the reliability of the distribution system is a variable. In (4.10), only one reliability will be selected by setting a Boolean $r_{s l o}^{S}$, such that $\sum_{o} r_{s l o}^{S}=1$ if $y_{s l}$ is selected, otherwise 0 . Finally, (4.11) ensures at least $r_{\text {TARGET, } l}$ is achieved on each load $l$, allowing it to be connected to multiple generators.

$$
\begin{array}{lr}
\sum_{o} r_{s l o}^{S} \leq y_{s l} & \forall s, \forall l \\
\sum_{S} \sum_{o} r_{s o}^{E} r_{s l o}^{S} \leq \ln \left(1-r_{\mathrm{TARGET}, l}\right) & \forall l
\end{array}
$$

Summarizing the linearization of the GS\&GLP step, (2.1) is converted in the linear combination of objectives of (4.1). Because (4.1) is still non-linear due to the product of variables, it is linearized by using discretization in (4.2). The constraints (4.3)-(4.8) are linear and ensure connectivity between generators and loads. Finally, the reliability constrains in (2.2)-(2.3) are linearized to (4.9) if distribution system's reliability is assumed as a lower boundary, or (4.10)-(4.11) if the distribution system's reliability is a variable to solve for. Constraints (4.9), (4.10)-(4.11) allows a load $l$ to be supplied by multiple generators, thus providing resiliency when a generator fails. In the next section the linearization of the formulations in the PDD step will be detailed.

\section{F. Power Distribution Design}

The PDD's objective in (3.1) is already linear, so the connectivity and reliability constraints can be considered. Power flow balance is enforced on every component $i \in \mathcal{N}$ by summing all incoming and outgoing power flows with its own generation power and load demand (if they exist). Incoming flows and load demand are considered positive, while outgoing flows and generation power are considered negative. Given the solution of the GS\&GLP step, each distribution path $k$ will have to satisfy the load demand $d_{k}^{L O A D}$ by supplying a generation power $d_{k}^{G E N}$. For the generator-load pair of the distribution path $k, d_{k}^{L O A D}=0$ in the generator, $d_{k}^{G E N}=0$ on the load, and $d_{k}^{L O A D}=d_{k}^{G E N}=$ 0 for the rest of the components forming the path. Incoming power flow from any component $j$ to component $i$ is 
denoted $P_{j i, k}$, while outgoing power flow from $i$ to any other component $j$ is denoted as $P_{i j, k}$ Flow balance constraint is then,

$$
\sum_{i \mid(j, i) \in \mathcal{A}} P_{j i, k}-\sum_{i \mid(i, j) \in \mathcal{A}} P_{i j, k}+d_{k}^{L O A D}-d_{k}^{G E N}=0 \quad \forall i \in \mathcal{N}, \forall k \in K
$$

For any components $i, j$ the power flow $P_{i j}^{k}$ flows in the $i \rightarrow j$ direction. However, it is possible to have flow in opposite direction by defining two variables for the same connection between $i, j$ (such that only one can be positive at once) allowing power flow to be reversed, e.g. recovering energy in regeneration modes. The connection from $i$ to $j$ can only be selected if these components have both been selected, then,

$$
\begin{array}{ll}
x_{i j} \leq x_{i} & \forall(i, j) \in \mathcal{A}, \forall i \in \mathcal{N} \\
x_{i j} \leq x_{j} & \forall(i, j) \in \mathcal{A}, \forall j \in \mathcal{N}
\end{array}
$$

Constraints (5.4)-(5.5) ensures that power $P_{i j, k}$ through any connection $i, j$ and power through any component $i$, can only flow if connection $x_{i j}$ or component $x_{i}$ have been selected. For the rest of the paper, the required flow along the distribution path $k$ is $d_{k}$, such that $d_{k}=\left|d_{k}^{L O A D}\right|$, for notation simplicity.

$$
\begin{array}{lr}
P_{i j, k} \leq d_{k} x_{i j} & \forall(i, j) \in \mathcal{A}, \forall k \in K \\
\sum_{j \in \mathcal{N}} P_{j i, k} \leq d_{k} x_{i} & \forall i \in \mathcal{N}, \forall k \in K
\end{array}
$$

The reliability constraint for the distribution system in (3.2) contains products and is non-linear, thus, logarithms can be used for linearization. Constraint (3.2) also requires a subset of connections and components to form the distribution path $k$. Due to the fact that connection $x_{i j}$ and component $x_{i}$ can be used for more than one distribution path $k$, Boolean variables $z_{i j, k}$ and $z_{i, k}$ are used to determine if $x_{i j}$ and $x_{i}$ are being used for distribution path $k$ once connection $x_{i j}$ and component $x_{i}$ have been selected respectively. Then,

$$
\begin{array}{ll}
z_{i, k} \leq x_{i} & \forall(i, j) \in \mathcal{A}, \forall k \in K \\
z_{i j, k} \leq x_{i j} & \forall(i, j) \in \mathcal{A}, \forall k \in K
\end{array}
$$

Similarly to (5.4)-(5.5), constraints (5.8)-(5.9) enforce no power flow (related to distribution path $k$ ) in component $i$ or connection $i, j$ if the component and connection have not been reserved for that distribution path $k$, hence,

$$
\begin{array}{lr}
P_{i j, k} \leq d_{k} z_{i j, k} & \forall(i, j) \in \mathcal{A}, \forall k \in K \\
\sum_{j \in \mathcal{N}} P_{j i, k} \leq d_{k} z_{i, k} & \forall i \in \mathcal{N}, \forall k \in K
\end{array}
$$


Now, assuming a single path (series system), the reliability constraint in (3.2) can be linearized as in (5.10) by taking the natural logarithm of both sides. The reliability of each distribution path $k$ must be at least equal to the reliability target $r_{\text {TARGET, } k}$.

$$
\sum_{j \mid(i, j) \in \mathcal{A}} z_{i j, k} \ln \left(r_{i j}\right)+\sum_{i \in \mathcal{N}} z_{i, k} \ln \left(r_{i}\right) \geq \ln \left(r_{\mathrm{TARGET}, k}\right) \quad \forall k \in K
$$

Lastly, (5.11) limits the power flow through connection $i, j$ and component $i$ to their maximum $P_{i j}^{\mathrm{MAX}}$ and $P_{i}^{\mathrm{MAX}}$.

$$
\begin{array}{lr}
0 \leq \sum_{k \in K} P_{i j, k} \leq P_{i j}^{\mathrm{MAX}} & \forall(i, j) \in \mathcal{A}, \forall k \in K \\
0 \leq \sum_{k \in K} P_{i, k} \leq P_{i}^{\mathrm{MAX}} & \forall i \in \mathcal{N}, \forall k \in K
\end{array}
$$

where $P_{i, k}=\sum_{j} P_{j i, k}$. The integrality $\{0,1\}$ on the Boolean variables used in this section can be written as,

$$
x_{i j}, x_{i}, z_{i j, k}, z_{i, k} \in\{0,1\} \quad \forall(i, j) \in \mathcal{A}, \forall i \in \mathcal{N}, \forall k \in K
$$

The PDD non-linear formulation of (3.1)-(3.2) has been converted into a MILP formulation in (3.1), (5.1)-(5.12). Other constraints can be added to this MILP formulation to satisfy specific needs, e.g. restrict components' power or cable sizes [44]. Given that (5.10) assumes a series system (single path) for the distribution path $k$, resiliency formulations will be introduced in order to provide multiple paths as proposed in (3.3). These approaches are known as resilient network designs [47].

\section{Resilient design for a set of failure scenarios}

If a failure event occurs in any component $i$ of the distribution path $k$, the single path breaks, the critical load is disconnected, and the reliability in (5.10) is no longer preserved. Therefore, a resilient design that produces a failure resistant system is required. Let $F$ be the set of failure scenarios comprising connection failures [47] and/or component failures [46] that the system is expected to survive. The EPDS must continue supplying critical loads if any of the failure scenarios of the set $F$ occurs (failure events in $F$ are assumed to be independent). Hence, the flow balance in (5.1) applies to every failure scenario. The connection flow $P_{i j, k}$ is now the maximum of all the power flows existing on every failure scenario $f$ of the set $F$, i.e. $P_{i j, k}=\max _{f} P_{i j, k}^{f}$, where $P_{i j, k}^{\mathfrak{f}}$ is the flow on connection $i, j$ for the failure scenario $f$ and distribution path $k$. Considering the set $F$ of failure scenarios, flow balance can be written as,

$$
\sum_{j \mid(i, j) \in \mathcal{A}} P_{j i, k}^{\natural}-\sum_{j \mid(j, i) \in \mathcal{A}} P_{i j, k}^{\mathfrak{f}}+d_{k}^{L O A D}-d_{k}^{G E N}=0 \quad \forall i \in \mathcal{N}, \forall k \in K, \forall f \in F
$$


Let $f_{i j}$ represent the existence of a failure in connection $x_{i j}$, then $f_{i j}=0$ for no failure, and $f_{i j}=1$ if failure occurs. Power flow along connection $i, j$ is guaranteed if, for the failure scenario $f$, this connection has not failed $\left(f_{i j}=0\right)$, otherwise, power flow is 0 , then,

$$
P_{i j, k}^{f} \leq d_{k} x_{i j}\left(1-f_{i j}\right) \quad \forall(i, j) \in \mathcal{A}, \forall k \in K, \forall f \in F
$$

Finally, constraint for $P_{i j, k}$ (maximum of all $P_{i j, k}^{f}$ ) is (6.3), and integrality on Boolean variables can be written in (6.4).

$$
\begin{array}{lr}
P_{i j, k}=\max _{f} P_{i j, k}^{f} & \forall(i, j) \in \mathcal{A}, \forall k \in K \\
x_{i j} \in\{0,1\} & \forall(i, j) \in \mathcal{A}
\end{array}
$$

The resilient network design for a set of failure scenarios is the MILP formulation (3.1), (6.1)-(6.4). It is possible to consider component selection by adding (5.2)-(5.5), and (5.11). Although the determination of an exhaustive failure set (all possible failures) could be cumbersome because of the large number of components/connections and simultaneous failure combinations, it is possible to determine a partial failure set, e.g. limited number of hazardous events that could compromise critical functions. At this point, it might be desirable to have an alternative if no set $F$ of failure scenarios could be determined. This situation could be found when considering disruptive designs with experimental technologies. In these cases, there is limited information on the failure modes of the system because technology can be at the prototype level (likely to happen in MEA). Hence, in addition to the resilient design for failure scenarios, a two-disjoint path formulation will be introduced.

\section{Resilient design with two-disjoint paths}

The distribution path $k$ has an alternate path to transfer power flow in case there is a failure on any component/connection that interrupts power supply. In this case, both paths are considered disjoint because the failure on one of them must not limit the capacity of the other. It is possible that the alternate path supplies power from a different generator or power source (auxiliary unit, storage device, etc.). In most cases, both paths do not share the same components and connections in order to manage failures on any component of the first (or second) path. This approach is known as the two-disjoint path problem [53]. Distjointness refers to the use of different connections and components for each path, such that the probability that at least one path survives is increased.

Let $q$ be the disjoint-path index, i.e. $q \in\{1,2\}$. When connection $i, j$ is selected, it can either allow power flow for the first or the second disjoint-path, thus $P_{i j, k}^{q=1}=0$ or $P_{i j, k}^{q=2}=0$. Similarly, when component $i$ is selected, it can either transfer power for the first or second disjoint-path, i.e. $\sum_{j \in N} P_{j i, k}^{q=1}=0$ or $\sum_{j \in N} P_{j i, k}^{q=2}=0$. Let the Boolean $u_{i j, k}$ and 
$u_{i, k}$ select which disjoint-path is the connection $i, j$ and component $i$ reserved for. The connection disjointness can be established as follows,

$$
\begin{array}{ll}
P_{i j, k}^{q=1} \leq d_{k} u_{i j, k} & \forall k \in K, \forall(i, j) \in \mathcal{A} \\
P_{i j, k}^{q=2} \leq d_{k}\left(1-u_{i j, k}\right) & \forall k \in K, \forall(i, j) \in \mathcal{A}
\end{array}
$$

Boolean $u_{i j, k}$ reserves power flow $P_{i j, k}^{q}$ for disjoint-path 1 or 2 only (not both) because either $u_{i j, k}=0$ or $1-u_{i j, k}=$ 0. Similarly, the component disjointness can be established as,

$$
\begin{array}{ll}
\sum_{j \in \mathcal{N}} P_{j i, k}^{q=1} \leq d_{k} u_{i, k} & \forall k \in K, \forall i \in \mathcal{N} \\
\sum_{j \in \mathcal{N}} P_{j i, k}^{q=2} \leq d_{k}\left(1-u_{i, k}\right) & \forall k \in K, \forall i \in \mathcal{N}
\end{array}
$$

Boolean $u_{i, k}$ reserves component's power flow for disjoint-path 1 or 2 (not both) because either $u_{i, k}=0$ or $1-$ $u_{i, k}=0$. Now, the power flow balance must be established for every disjoint-path $q$, thus, it can be written as:

$$
\sum_{j \mid(i, j) \in \mathcal{A}} P_{i j, k}^{q}-\sum_{j \mid(j, i) \in \mathcal{A}} P_{j i, k}^{q}+d_{k}^{L O A D}-d_{k}^{G E N}=0 \quad \forall i \in \mathcal{N}, \forall k \in K, q \in\{1,2\}
$$

If $x_{i j}$ is set, $P_{i j}^{k, p}$ is allowed to flow on either disjoint-path according to (7.4).

$$
P_{i j, k}^{q} \leq d_{k} x_{i j} \quad \forall k \in K, \forall(i, j) \in \mathcal{A}, q \in\{1,2\}
$$

The integrality constraints on the Boolean $u_{i j, k}$ and $u_{i, k}$ are shown below.

$$
u_{i j, k}, u_{i, k} \in\{0,1\} \quad \forall k \in K, \forall(i, j) \in \mathcal{A}, \forall i \in \mathcal{N}
$$

The reliability of two disjoint paths can be obtained using the probability of the occurrence of any two events, that is $\mathrm{P}(a \cup b)=\mathrm{P}(a)+\mathrm{P}(b)-\mathrm{P}(a \cap b)$, and for independent events $\mathrm{P}(a \cap b)=\mathrm{P}(a) \mathrm{P}(b)$. Let $r_{k}^{q}$ be the reliability of the disjoint-path $q$, then, the probability that at least one path survives must at least reach target $r_{\text {TARGET, } k}$,

$$
r_{k}^{q=1}+r_{k}^{q=2}-r_{k}^{q=1} r_{k}^{q=2} \geq r_{\mathrm{TARGET}, k} \quad \forall k \in K
$$

For the calculation of each disjoint-path's reliability $r_{k}^{q},(5.10)$ suffices (reliability of a single path, series system). Considering (5.10), the product of the disjoint-paths' reliabilities in (7.6) produces an extensive series of products between variables. In order to avoid performing linearization on each of these products, a simpler approach to represent the disjoint-path's reliability is introduced. Let a set of variables $s_{i, k}^{q}$ be defined as the probability of power successfully reaching component $i$ (starting at the generator) when component $i$ is reserved for disjoint-path $q$ (recall that distribution path $k$ consists in two disjoint-paths). Then, $s_{i, k}^{q}$ is,

$$
s_{j, k}^{q} \leq r_{i j} r_{i} s_{i, k}^{q}+\left(1-u_{i j, k}\right) \quad \forall k \in K, q \in\{1,2\}, \forall(i, j) \in \mathcal{A}
$$


If component $i$ is selected, $u_{i j, k}=1$ and $s_{j, k}^{q} \leq r_{i j} r_{i} s_{i, k}^{q}$; otherwise (7.7) relaxes $s_{j, k}^{q}$ to an arbitrary value if component $i$ is not selected, i.e. $u_{i j, k}=0$. For the generators, $s_{j, k}^{q}$ is,

$$
s_{G E N, k}^{q}=1 \quad \forall k \in K, q \in\{1,2\}
$$

When (7.7) is applied to the load of the distribution path $k, s_{j, k}^{q}$ is the probability of successfully reaching the load and $s_{j, k}^{q}$ can be written as $s_{L O A D, k}^{q}$. Then $s_{L O A D, k}^{q}$ is the reliability of the disjoint-path $q$, so that $s_{L O A D, k}^{q}=r_{k}^{q}$. Now, considering the variables $s_{L O A D, k}^{q}$, the constraint on the reliability of the two disjoint-paths (7.6) can be rewritten as,

$$
s_{L O A D, k}^{q=1}+s_{L O A D, k}^{q=2}-s_{L O A D, k}^{q=1} s_{L O A D, k}^{q=2} \geq r_{\mathrm{TARGET}, k} \quad \forall k \in K
$$

The values of $s_{L O A D, k}^{q=1}$ and $s_{L O A D, k}^{q=2}$ in (7.9) could be identical. This could originate an algorithmic issue known as symmetry problem, which can produce high computational effort during MILP optimization. To break the potential symmetry problem in (7.9), an additional constraint is introduced to speed up MILP optimization,

$$
s_{L O A D, k}^{q=1} \geq s_{L O A D, k}^{q=2} \quad \forall k \in K
$$

With this approach, only one product in (7.9) requires linearization (unlike the extensive series of products in (7.6)). An alternative to the linearization of $s_{L O A D, k}^{q=1} s_{L O A D, k}^{q=2}$ is to express (7.9) as a group of linear constraints that select a value for the left hand side of (7.9) directly. Allow each $s_{L O A D, k}^{q}$ to be discretized in a vector containing the disjointpath' reliability values, i.e. $\boldsymbol{\alpha}=\left[\alpha_{1} \ldots \alpha_{a}\right]$ and $\boldsymbol{\beta}=\left[\beta_{1} \ldots \beta_{b}\right]$ for $q=1$ and $q=2$ respectively. Also, allow a matrix $\Gamma[a \times b]$ have elements $\gamma_{a, b}$ such that $\gamma_{a, b}=\alpha_{a}+\beta_{b}-\alpha_{a} \beta_{b}$. Let a Boolean $\sigma_{a b, k}$ select a unique value for $\gamma_{a, b}$, then, constraint (8.1) ensures that only one element $\gamma_{a, b}$ from the matrix $\Gamma$ is selected

$$
\sum_{a} \sum_{b} \sigma_{a b, k}=1 \quad \forall k \in K
$$

The two path reliability constraint in (7.9) can be written as,

$$
\sum_{a} \sum_{b} \gamma_{a, b} \sigma_{a b, k} \geq r_{\mathrm{TARGET}, k} \quad \forall k \in K
$$

A condition for (8.1) and (8.2) is that a unique value for each disjoint path's reliability ( $\alpha_{a}$ and $\left.\beta_{b}\right)$ must be selected. For the disjoint path $q=1$, such a condition can be written as,

$$
\sum_{a} \sum_{b} \alpha_{a-1} \sigma_{a b, k} \leq 8_{L O A D, k}^{q=1} \leq \sum_{a} \sum_{b} \alpha_{a+1} \sigma_{a b, k} \quad \forall k \in K
$$

Similar constraint for $q=2$ can be written by replacing $\alpha_{a}$ with $\beta_{b}$ and $s_{L O A D, k}^{q=1}$ with $s_{L O A D, k}^{q=2} ; \alpha_{a-1}$ and $\alpha_{a+1}$ act as an upper and lower values for $\alpha_{a}$. A drawback of this approach is the large number of $\sigma_{a b, k}$ variables if higher accuracy 
were required. In (8.2), $\gamma_{a, b}$ could be stuck around $r_{\text {TARGET, } k}$ even when the disjoint paths' reliabilities are higher. To prevent this, an additional term in the optimization objective is needed to push $s_{L O A D, k}^{q}$ to its actual value. This term should be comparatively small with respect to total cost to avoid biasing cost optimization. Then,

$$
\min _{P, x, s, u} \sum_{(i, j) \in \mathcal{A}}\left(c_{i j} x_{i j}+c_{\mathrm{kW}}^{i j}\left(\sum_{k \in K} P_{i j, k}\right)\right)+\sum_{i \in \mathcal{N}}\left(m_{i} v_{i}+m_{\mathrm{kW}}^{i}\left(\sum_{k \in K} P_{i, k}\right)\right)-\sum_{k \in K} \sum_{q} s_{L O A D, k}^{q}
$$

\section{Briefing of the MILP resilient design formulations}

This section concludes the MILP reliability based network design formulations for the synthesis of MEA EPDS architectures following a PBD-inspired framework. The EPDS design has been split into two steps: GS\&GLP and PDD. In the GS\&GLP, a group of power sources or generators are selected, sized, and assigned to the loads. Then, this optimum is used to synthesize a topology for the EPDS in the PDD step. In order to increase power availability in the loads, resiliency was introduced in the MILP formulations of GS\&GLP and PDD. In PDD, resiliency was appointed by designing an EPDS that tolerates the occurrence of failures, either by preparing the system for a set of pre-defined failure scenarios or providing two-disjoint paths for every distribution path. Specialized MILP solver packages will be used shortly to synthesize a MEA EPDS architecture in the case study of the next section.

\section{Case Study}

The purpose of this case study is to exemplify the design framework presented in Section III by applying the reliability-based MILP formulations of Section V in the synthesis of a MEA EPDS architecture. Although there are an important number of MEA applications (electric propulsion, turbo-electric, etc.) and EPDS network structures (DC, AC, AC \& DC, single bus, ring, etc.), we provide a complete design assessment for a small-aircraft MEA DC EPDS architecture so that the designer can perceive the potential of our design framework proposal for other applications. The technical considerations for the MEA EPDS design are detailed below.

- The MEA EPDS structure will be based on a DC network with two voltage levels: a high-voltage (HV) level and a low-voltage (LV) level, because there are HV and LV loads. In its simplest structure, there are a number of source matrix contactor buses (bus bars) that distribute generation power, a number of power converters to transform power from HV to LV power, and a number of LV buses. This network structure has been studied in several publications as promising MEA EPDS [4], [16], [54]. Hence, following the design 
framework presented in this paper, the templates $\mathcal{G}=\{g, \mathcal{E}\}$ for the GS\&GLP step and $\mathcal{G}=\{\mathcal{N}, \mathcal{A}\}$ for the PDD step are shown in Fig. 5.
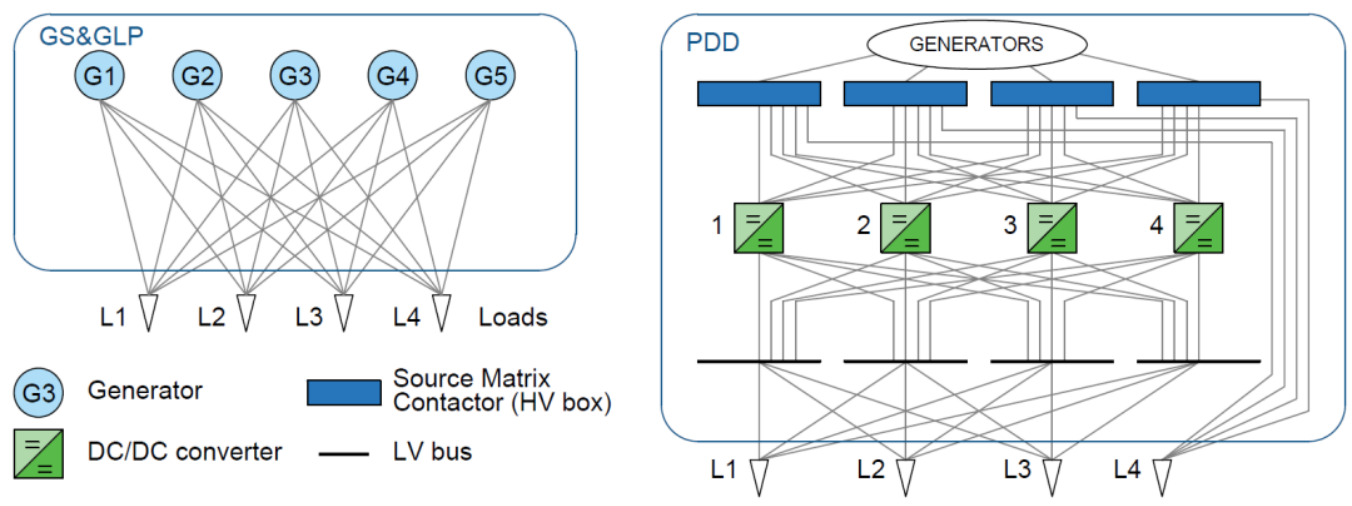

Fig. 5 Templates for GS\&GLP step (left), and PDD step (right)

- A PMSM generator with a power density of $7.0 \mathrm{~kW} / \mathrm{kg}$ is assumed to be available. This assumption is in accordance with the expected technology for low carbon MEA propulsion [55]. The number of generators will be arbitrarily limited to 5 considering the possibility of multiple small generators.

- A power density of $6.7 \mathrm{~kW} / \mathrm{kg}$ will be used for power electronic conversion [55] (assuming high power density SiC-based power electronics technology is available). The weight of power conversion is assumed to be proportional to the power flow transferred, and this flow is assumed to be proportional to the cost.

- The case study is solved using a Windows High Spec PC Intel Xeon 64-bit 3.60GHz running CPLEX Studio IDE 12.9.0 [56].

The MEA EPDS supplies power to a group of 4 loads which total demand is $125 \mathrm{~kW}$. The loads' requirements (power demands and reliabilities) are shown in Table 1 . This table is part of the initial design requirements. The reliability target is expressed as a probability, e.g. load $L_{1}$ requires to be supplied (with generation power) with a probability of at least $1-\left(1 \times 10^{-9}\right)$. Loads $L_{1}$ to $L_{3}$ are LV DC (low voltage), and $L_{4}$ is the only HV DC load (high voltage).

Table 1 Design requirements for on-board loads (Part I)

\begin{tabular}{clcccc}
\hline \multirow{3}{*}{ Loads } & & $L_{1}$ & $L_{2}$ & $L_{3}$ & $L_{4}$ \\
\cline { 3 - 6 } & $L_{l}[\mathrm{~kW}]$ & 50 & 25 & 40 & 10 \\
& $1-r_{\mathrm{TARGET}, l}$ & $1.0 \times 10^{-9}$ & $1.0 \times 10^{-6}$ & $1.0 \times 10^{-6}$ & $1.0 \times 10^{-6}$ \\
\hline \hline
\end{tabular}


The reliability targets in Table 1 are accompanied by a set of functional specifications, in this case, resiliency requirements. In addition to setting $L_{1}$ as the only critical load because it has the lowest probability of failure (of not being supplied) and determine the optimal MEA EPDS architecture for this application, we propose an extended set of resiliency requirements in Table 2 to illustrate the potential of the design framework.

Table 2 Design requirements for on-board loads (Part II)

\begin{tabular}{|c|c|c|c|c|c|c|c|c|c|c|c|c|c|c|}
\hline Scenario & 1 & 2 & 3 & 4 & 5 & 6 & 7 & 8 & 9 & 10 & 11 & 12 & 13 & 14 \\
\hline Formulation & Single path & \multicolumn{6}{|c|}{ Design for a Failure Set } & \multicolumn{7}{|c|}{ Two Disjoint Path } \\
\hline $\begin{array}{r}\text { Critical } \\
\text { loads }\end{array}$ & N/A & All & 1,3 & 1,3 & 1,3 & 4 & 1,4 & All & 1,3 & 1,4 & 4 & 2,3 & 2,4 & 1 \\
\hline $\begin{array}{r}\text { Failure set } \\
\qquad F\end{array}$ & - & $\begin{array}{l}\text { Conv. } \\
\text { \& HV } \\
\text { box }\end{array}$ & Conv. & $\begin{array}{l}\text { Conv. } \\
\& \text { LV } \\
\text { bus }\end{array}$ & $\mathrm{N}-1$ & $\begin{array}{l}\text { HV } \\
\text { box }\end{array}$ & $\begin{array}{l}\text { Conv. } \\
\text { \& HV } \\
\text { box }\end{array}$ & - & - & - & - & - & - & - \\
\hline $\begin{array}{r}\text { MILP } \\
\text { Optimization } \\
\text { objective }\end{array}$ & (3.1) & \multicolumn{6}{|c|}{ (3.1) } & \multicolumn{7}{|c|}{ (9) } \\
\hline $\begin{array}{r}\text { MILP } \\
\text { Constraints }\end{array}$ & $\begin{array}{l}(5.1) \text { to } \\
(5.12)\end{array}$ & \multicolumn{6}{|c|}{$\begin{array}{l}\text { Critical loads: }(6.1) \text {, to }(6.4) \\
\text { Non critical loads: }(5.1) \text { to }(5.12)\end{array}$} & \multicolumn{7}{|c|}{$\begin{array}{c}\text { Critical loads: (7.1) to (7.5), (7.7)-(7.8), (7.10), (8.1)-(8.3) } \\
\text { Non critical loads: (5.1) to (5.12) }\end{array}$} \\
\hline
\end{tabular}

There are 13 additional scenarios to the single path formulation (scenario 1). The scenarios 2-7 require some loads to be critical and the distribution system to be resilient over a set of failures, e.g. scenario 4 defines load 1 and 3 as critical and its EPDS must be prepared for single failures in any power converter or LV buses. In the case of scenario 5, N-1 means that the EPDS is resilient under any single failure of any component. Likewise, the scenarios 8-14 require some loads to be critical, but for those critical loads there will be two-disjoint paths to be supplied while the non-critical loads are supplied through single paths. The MILP optimization objective and constraints in Table 2 refer to the optimization formulations of the PDD step. To begin with, the GS\&GLP step is performed shortly.

\section{Generator selection and generator-load pairing}

The generator's rating power is in the range $25 \mathrm{~kW}-150 \mathrm{~kW}$ (a small aircraft has $\sim 300 \mathrm{~kW}$ per engine) and its loading will be restricted to $40 \%$ minimum to avoid very low efficiencies. The weight of the generators is inversely proportional to the power density, then $w_{s} \propto \frac{1}{7 \mathrm{~kW} / \mathrm{kg}}$ or $w_{s} \propto 0.1428 P_{s}^{G}$. An efficiency function $\eta_{s}$ relating power rating $P_{s}^{G}$ and loading $\beta_{s}$ is approximated to be able to select generators of different sizes and loadings. The generator's cost to weight ratio is $870 £ / \mathrm{kg}$ and a reliability of $1-1.0 \times 10^{-5}$ is considered for all the generators. The power distribution reliability is considered to be in the range of $0.9000-0.9997$. The power generation system design specifications for the GS\&GLP step (generation system platform abstraction) are tabulated in Table 3. 
Table 3 Power Generation System platform abstraction for GS\&GLP

\begin{tabular}{|c|c|c|}
\hline \multirow{7}{*}{ Generators } & $P_{s}^{G}[\mathrm{~kW}], \beta_{s}[$ p.u. $]$ & $P_{s}^{M I N}=25, P_{s}^{M A X}=150 ; \quad \beta_{s}^{M I N}=0.4, \beta_{s}^{M A X}=1.0$ \\
\hline & $w_{s}\left(P_{S}^{G}\right)[\mathrm{kg}]$ & $12.2+0.1428 P_{s}^{G}$ \\
\hline & $\eta_{s}\left(\beta_{s}\right)[\%]$ & $\begin{array}{c}22.09+0.2563 P_{s}^{G}+95.23 \beta_{s}-0.0004374\left(P_{s}^{G}\right)^{2}-0.1324 P_{s}^{G} \beta_{s}-40.46 \beta_{s}{ }^{2} \\
\text { Approximated function for values of } P_{s}^{G} \in\left[P_{s}^{M I N}, P_{s}^{M A X}\right]\end{array}$ \\
\hline & $\eta_{s}^{\mathrm{LOSS}}\left(P_{S}^{G}, \beta_{S}\right)[\%]$ & $P_{s}^{G} \beta_{s}\left(100\left(\eta_{s}^{-1}\left(\beta_{s}\right)\right)-1\right)$ \\
\hline & Cost (given $w \propto c$ ) & $870 £ / \mathrm{kg}$ \\
\hline & $1-r_{s}$ & $1.0 \times 10^{-5}$ \\
\hline & $r_{V S}$ & or $1-r_{V S}:\left[1.0 \times 10^{-1}, 3.0 \times 10^{-4}\right]$ \\
\hline
\end{tabular}

The GS\&GLP problem is solved considering the load requirements of Table 1 and the platform abstractions of Table 3. The optimization objective of the GS\&GLP is (4.2) (with weighting coefficients $a_{1}=a_{2}$ ), the connectivity constraints are (4.3)-(4.8), and the reliability constraints (resiliency) are (4.10)-(4.11). Then, in simplified notation,

$$
\begin{gathered}
\left.\min \sum_{\begin{array}{c}
s \\
\text { subject to: }
\end{array}}\left(a_{1} \text { (generator weight }\right)+a_{2}\left(\text { generator } \operatorname{loss}_{s}\right)\right) \\
\text { (4.3) to (4.8), (4.10) to (4.11) }
\end{gathered}
$$

The optimization selects a group of generators (with minimum weight and losses) such that connectivity and resiliency constraints are satisfied. The results of the GS\&GLP step are presented in Table 4. Three generators out of five were selected and the total generation capacity is $300 \mathrm{~kW}$. Also, 9 out of 20 distribution paths were selected. Given that the reliability requirements of the loads (Table 1) are high, all loads are connected to two generators at least $\left(L_{1}\right.$ is connected to all 3 generators), so generation capacity is more than double.

\begin{tabular}{|c|c|c|c|c|}
\hline & $\bar{G}_{1}$ & $\bar{G}_{2}$ & $\overline{G_{3}}$ & $G_{5}, G_{6}$ \\
\hline$P_{S}^{G}(\mathrm{~kW})$ & 125 & 50 & 125 & Not selected \\
\hline Loads served & $\left\{L_{1}, L_{2}, L_{3}, L_{4}\right\}$ & $\left\{L_{1}\right\}$ & $\left\{L_{1}, L_{2}, L_{3}, L_{4}\right\}$ & -- \\
\hline Set $K$ & $y_{11}, y_{12}, y_{13}, y_{14}$ & $y_{21}$ & $y_{31}, y_{32}, y_{33}, y_{34}$ & -- \\
\hline $\begin{array}{r}\text { Reliability } r_{s l} \text { of } \\
\text { the distribution } \\
\text { path } k\end{array}$ & $\begin{array}{l}1-r_{11}=1.6 \times 10^{-3} \\
1-r_{12}=1.6 \times 10^{-3} \\
1-r_{13}=6.0 \times 10^{-4} \\
1-r_{14}=6.0 \times 10^{-4}\end{array}$ & $1-r_{21}=1.0 \times 10^{-3}$ & $\begin{array}{l}1-r_{31}=6.0 \times 10^{-4} \\
1-r_{32}=6.0 \times 10^{-4} \\
1-r_{33}=1.6 \times 10^{-3} \\
1-r_{34}=1.6 \times 10^{-3}\end{array}$ & -- \\
\hline
\end{tabular}

\section{Table 4 GS\&GLP solution for a MEA EPDS design}

The optimal solution of the GS\&GLP is used in the PDD step to refine the distribution paths (build a power distribution topology). The reliabilities $r_{s l}$ of each path are used as reliability targets in the PDD step. 


\section{Power Distribution Design}

Recall that there are three types of distribution components considered in the EPDS network structure: HV Source Matrix Contactor (HV box), HV/LV DC converters, and LV DC bus. The Source Matrix Contactor (HV box) allows power generation transfer without cross-connecting generators. The HV/LV DC converters are assumed to be of the dual active bridge type (DAB) and can be connected to more than component in both sides. The LV buses can distribute power to multiple loads from more than one HV/LV DC converter. The power density ( $p$. d.), weight $\left(w_{i j}, w_{i}\right)$, fixed costs $\left(c_{i j}, m_{i}\right)$, variable costs $\left(c_{i j}^{\mathrm{kW}}, m_{i}^{\mathrm{kW}}\right)$ reliability $\left(r_{i}, r_{i j}\right)$, and maximum power for all connections $i, j$ and all type of components is shown in Table 5 . The power density of the HV/LV converters is $6.7 \mathrm{~kW} / \mathrm{kg}$ as previously stated. Although the characteristics for the connections $i, j$ require the definition of voltage values, conductor types, insulation types, etc., the values shown in Table 5 are assumed for illustration purposes.

Table 5 Power Distribution platform abstraction for PDD

\begin{tabular}{clcccc}
\hline \hline & & HV DC sw. box & Converters & LV DC buses & Connections $(i j)$ \\
\cline { 3 - 6 } Power & $p . d .[\mathrm{kW} / \mathrm{kg}]$ & 8.0 & 6.7 & 14.5 & 25.5 \\
distribution & $w_{i}, w_{i j}[\mathrm{~kg}]$ & 1.8 & 8.5 & 1.8 & 0.2 \\
& $m_{i}^{\mathrm{kW}}, c_{i j}^{\mathrm{kW}}[\mathrm{kE} / \mathrm{kW}]$ & 0.1 & 0.3 & 0.1 & 0.05 \\
\cline { 2 - 6 } & $m_{i}, c_{i j}[\mathrm{k} f]$ & 12 & 48 & 12 & 1 \\
& $r_{i}, r_{i j}$ & $5.0 \times 10^{-6}$ & $1.0 \times 10^{-4}$ & $5.0 \times 10^{-6}$ & $2.0 \times 10^{-6}$ \\
\cline { 2 - 5 } & $P_{i}^{\mathrm{MAX}}, P_{i j}^{\mathrm{MAX}}[\mathrm{kW}]$ & 500 & 800 & 500 & 1000 \\
\hline \hline
\end{tabular}

Considering the optimal solution of the GS\&GLP step shown in Table 4, the MILP formulations presented in Section V.D are used to synthesize a MEA EPDS architecture considering the platform abstractions of Table 5. Several scenarios (consisting in different design requirements) were proposed in Table 2 and these options are explored in this case study for the synthesis of resilient distribution architectures.

The set $K$ of distribution paths connecting generators and loads (GS\&GLP optimal solution in Table 4) are divided into two subsets: one that contains generators supplying non-critical loads $\left(K_{N C}\right)$, and other that contains generators supplying critical loads $\left(K_{C}\right)$. Therefore, connectivity and resiliency constraints applied on distribution paths differ depending on the critical load combination, as shown in the scenarios of Table 2 . The power flow $P_{i j}$ in any connection $i, j$ consists in the summation of the power flows from distribution paths supplying critical and non-critical loads. 
Scenario 1 (single path) results in the EPDS's topology of Fig. 6(a). The generators supply the LV DC loads by the series system HV box - HV/LV Converter - LV bus, in other words, each distribution path supplying LV DC loads use the same series system ( $L_{4}$ is supplied by HV box). Fig. 6(b) illustrates the distribution paths (set $K$ ).

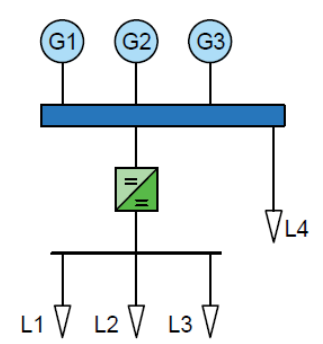

(Sc. 1)
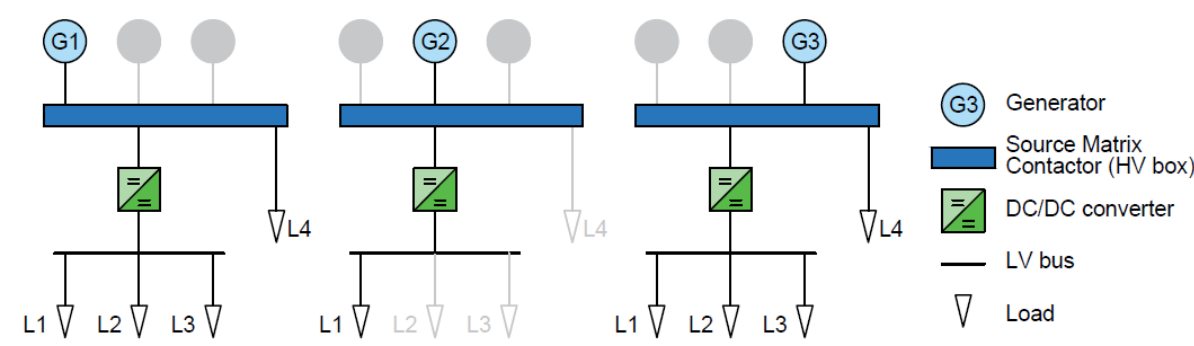

(distribution paths visualization)

Fig. 6 MEA EPDS architecture with single path formulation or Scenario 1 (a) EPDS; (b) visualization of the distribution paths (generator-load pairs) of Table $4\left(G_{1}\right.$ to $\left\{L_{1}, L_{2}, L_{3}, L_{4}\right\}, G_{2}$ to $\left\{L_{1}\right\}, G_{3}$ to $\left.\left\{L_{1}, L_{2}, L_{3}, L_{4}\right\}\right)$

Note that if a failure occurred in any component of the EPDS in Fig. 6(a), power supply to LV DC loads is cut. Given the lack of alternative paths to distribute power to the loads in the Scenario 1 in Fig. 6, resilient designs are used. The resilient designs for a failure set corresponds to the formulations in Scenarios 2 to 7 of Table 2, while the resilient designs with two disjoint paths-corresponds to the formulations in Scenarios 8 to 14 .

The EPDS's topologies that result from the synthesis of resilient designs for a failure set (Scenarios 2 to 7 of Table 2) are shown in Fig. 7 (critical loads are solidly colored, non-critical loads have no fill). Each failure case is determined by row Failure set $F$ of the corresponding column of Table 2. Conv., HV box, and LV bus refer to single failures in power converters, HV boxes, and LV buses respectively. The failures are modelled by determining which connections $i j$ are active and which are not. Each case of the set $F$ has $48 f_{i j}$ elements (there are 48 possible connections $x_{i j}$ according to template $\mathcal{G}$ in Fig. 5) such that $f_{i j}=1$ for failed connection $i, j$. Then, a failure in a component $i$ is determined by setting all incoming and outgoing connections as failed, that is, $f_{j i}=f_{i j}=1$ for all the components $j$ that are connected to component $i$. Depending on the set $F$, the number of selected components varies.

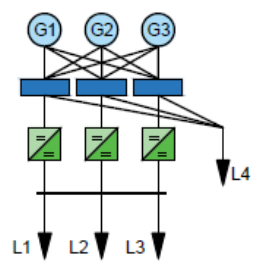

(Sn. 2)

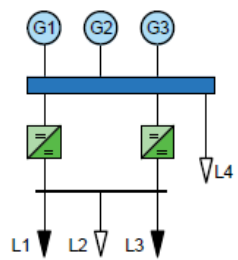

(Sn. 3)

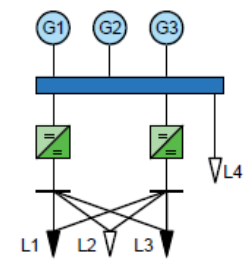

(Sn. 4)

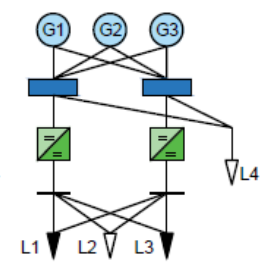

(Sn. 5)

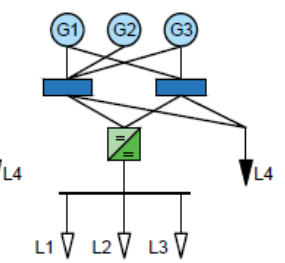

(Sn. 6)

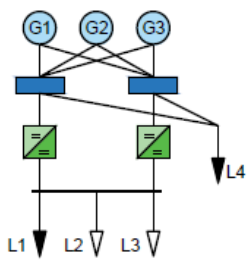

(Sn. 7)

Fig. 7 Optimal MEA EPDS architecture for resilient requirements (failure-resistant) 
In Scenario 2, the failure set $F$ contains 8 possible failure in distribution components: single failure on $\mathrm{HV} / \mathrm{LV}$ converters ( 4 in total), and single failure on HV boxes ( 4 in total). That is why it has the largest number of HV boxes and $\mathrm{HV} / \mathrm{LV}$ converters. In all cases where failure on HV/LV converter is considered (Scenarios 2-7 except 6), the EPDS topology has at least 2 converters (redundant converters). The scenarios where failures on HV box are not considered (3 and 4), there is only one HV box (see Fig. 7(b) and (c)). Scenario 2 in Fig. 7(a) (all loads are critical) is the heaviest and most expensive option, yet there is only one LV bus because failures in LV buses where not considered. The lightest (cheapest) EPDS is the Scenario 6 (Fig. 7(e)) which considers failures in HV boxes only. This EPDS is appropriate if the HV loads ( $L_{4}$ in this case) are the only critical loads. In the cases where LV loads are critical, ( $L_{1}$ and $L_{3}$ on Scenarios 2 to 5 and 7), weight and cost can be reduced with respect to the topology in Fig. 7(a). In general, some weight (and cost) savings are possible depending on the critical load combination and the predefined failure set $F$ (different design requirements).

When a failure set cannot be determined, a resilient design with two disjoint-path formulations for critical loads can be used. The MEA EPDS topologies synthesized with two disjoint path formulations are presented in Fig. 8 .

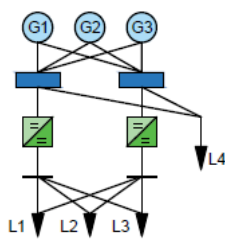

(Sn. 8)

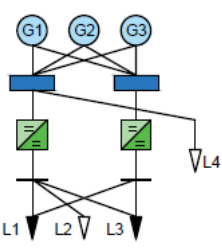

(Sn. 9)

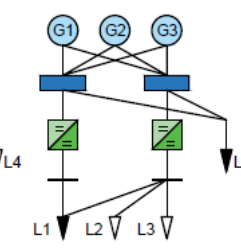

(Sn. 10)

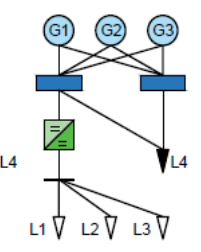

(Sn. 11)

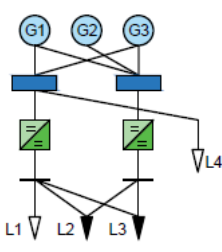

(Sn. 12)

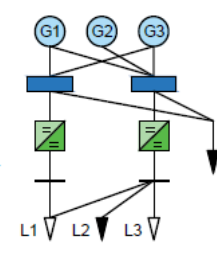

(Sn. 13)

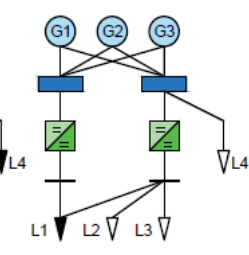

(Sn. 14)

Fig. 8 Optimal MEA EPDS architecture for resilient requirements (two-disjoint path)

The two disjoint-path formulation allows the synthesis of an EPDS architecture consisting of two components of each type in all Scenarios of Fig. 8 (except Scenario 11 in Fig. 8(d)) because there is at least one LV load that is critical. The disjointness caused by $u_{i j, k}, u_{i, k}$ in (7.1)-(7.2) allows the existence of separate distribution paths, i.e. two paths of the form HV box-HV/LV converter-LV bus for the critical LV loads, and two paths with different HV boxes to supply critical HV loads (only where $L_{4}$ is critical, Scenarios $8,10,11$, and 13 ). The reliability $8_{L O A D, k}^{q}$ of each path has been determined with the linear transformation of (8.1)-(8.3). Similarly to the architectures in Fig. 7, some critical load combinations allow weight and cost savings without compromising the resiliency of the system. The lightest (cheapest) option can be obtained by setting HV loads as the critical loads, as illustrated in Fig. 8(d) (Scenario 11). For the rest of the cases where LV loads are critical, the system's cost is increased. 
A summary of the results in terms of cost, weight, efficiency -losses-, and reliability for each of the EPDS Scenarios of Table 2 is presented in Table 6 . This table also tabulates the solving time for comparison purposes. Additionally, a graphical comparison to illustrate the potential of the proposed design framework to elaborate trade-off analysis between cost, weight and reliability for all scenarios of Table 2 is presented in Fig. 9. For each scenario, there is a line that connects the largest and lowest reliability provided by the optimal architecture.
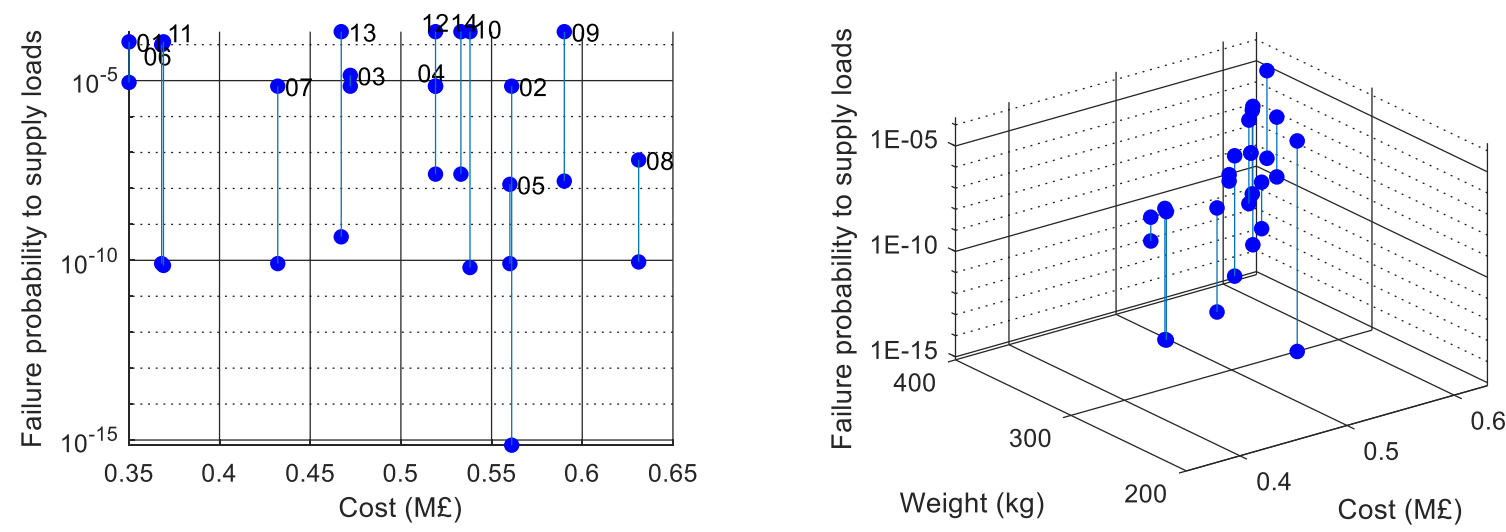

Fig. 9 Trade-off between cost, weight, and reliability for optimal MEA EPDS architectures

Given that cost is considered proportional to weight, the main trade-off analysis can be conducted with the cost vs. reliability (failure probability to supply loads) of Fig. 9. For instance, below a cost of $\sim 500 \mathrm{k} £$, a MEA EPDS architecture is unable to achieve reliabilities below $1-1 \times 10^{-11}$. However, most of the solutions provide at least a reliability between $1-1 \times 10^{-8}$ and $1-1 \times 10^{-4}$ in a specific load terminal. Based on the results presented in Table 6 and Fig. 9, the following conclusive remarks apply:

- In all cases, a minimum reliability level was achieved, i.e. $r \geq r_{\mathrm{TARGET}, l}$ as required by (1.3).

- Although Scenario 1 achieves a minimum reliability at a minimum cost, no resiliency is provided.

- In general, the EPDSs synthesized to overcome failures from a failure set have higher reliabilities than the EPDSs synthesized with two disjoint-path formulations.

- The EPDSs built with two disjoint-paths have slightly higher costs compared to EPDSs synthesized from resilient designs for a failure set.

- Scenarios 6, 11, and 13 are the most economical because the HV load $\left(L_{4}\right)$ is set to be critical. HV loads requires no power conversion unlike LV loads which requires at least one power conversion stage. 
- $\quad$ Scenarios $7,3,4,5$, and 9 are the most economical in the case that a set of LV loads $\left(L_{1}\right.$ and $\left.L_{3}\right)$ are critical. The costs increase due to the power conversion stage required.

- Scenario 14 has one critical LV load $\left(L_{1}\right)$ and its topology's cost is slightly less expensive than having two critical LV loads including $L_{1}$, as in Scenario 9. However, the lower the power requirements of the LV loads, the lower the cost is, as can be seen from the results of Scenario 12.

- The solving times of the resilient designs for a failure set are higher (on average) than the resilient designs with two-disjoint paths designs. This is due to the higher number of constraints per failure case.

Lastly, there are number of opportunities with the optimal MEA EPDS architectures of Fig. 7 and Fig. 8. Because these optimal architectures were originated from different requirements established on Table 2, there is no further selection of an optimum but a careful assignment of any of these architectures to a MEA application that needs a specific performance. For instance, the topology of scenario 6 suits best when an HV load is critical and all the LV demand can be shed. The optimal architectures found in this paper resemble the results found in [10], [57] (topology optimization) and correspond to similar network structures found in [4], [16], [54]. However, the design framework presented allows the designer explore a design space and investigate the optimum EPDS for a set of requirements, or even perform a trade-off analysis for several sets of requirements, as evidenced in Table 6.

In conclusion, the reliability based MILP network design formulations of Section V have been used to synthesize a MEA EPDS architecture for a small aircraft application. The design framework comprises two steps: GS\&GLP and PDD. Although single path formulations complies with a minimum reliability, resilient formulations enable the EPDS to route power from generators to loads through alternate paths. Resilient designs for a failure set or two disjoint-path on critical loads were formulated. In addition to the load requirements, the EPDS architecture also depends on the resilient requirements and the critical load combination. Hence, an optimal EPDS's architecture can fit a MEA application depending on the requirements. As a design exercise, Table 6 presents a solution palette for different scenarios of the case study. Loads that require no power conversion stages will contribute in having a lighter and more economical MEA EPDS, unlike the LV loads which add a higher number of components and consequently require a heavier (and costlier) EPDS. The design formulations presented in this paper allows the synthesis of more complex architectures, e.g. considering energy storage, different levels of load criticality, AC and DC conversions, several levels of power conversion, etc. Further refinement steps for defining distribution component technology selection and sizing will be explored in future research works. 
Table 6 Case study results for the MEA EPDS architectures of scenarios on Table 2

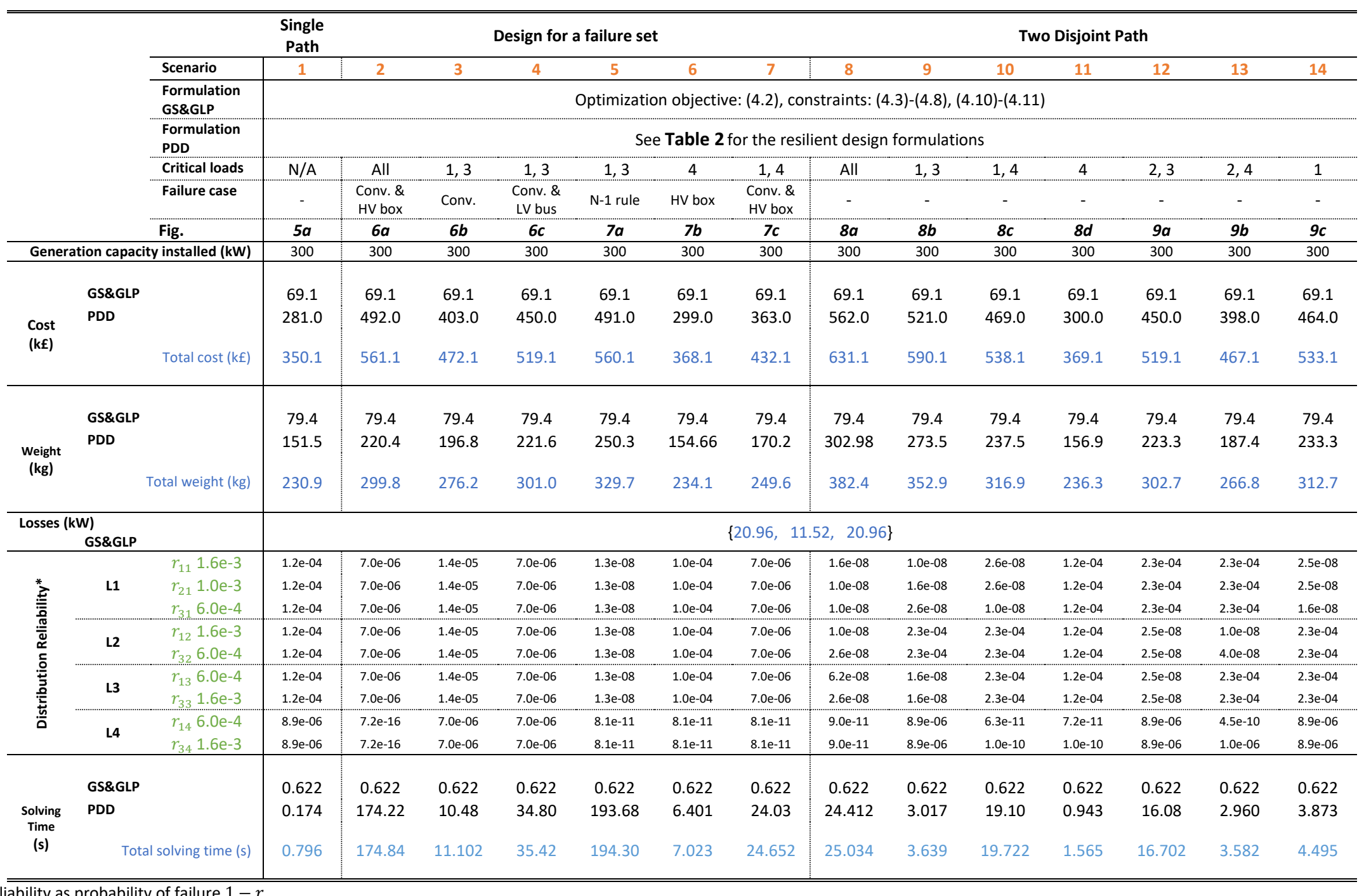

* Reliability as probability of failure $1-r$ 


\section{Conclusion}

The synthesis of a MEA EPDS architecture has been proposed by employing a PBD-inspired design framework that relies on reliability based MILP network design formulations. The proposed design approach allows handling of the design's complexity by dividing the architecture synthesis in two steps, Generator Selection \& Generator-Load Pairing and Power Distribution Design, supported by the relation between weight, cost, efficiency, and reliability for aircraft electrical systems. These steps are solved sequentially using linearization techniques and MILP formulations that model resiliency in an efficient manner in order to satisfy the design's reliability requirements. Resiliency is formulated as a group of constraints that ensure EPDS power availability in critical loads, either surviving a failure set or assigning two disjoint-paths for critical loads. A case study for a MEA application has shown that the optimal EPDS depends on the resiliency requirements and the critical load combination, from which several optimum architectures can be appropriately assigned to a MEA application that requires such performance and in some cases weight and cost reductions are possible. Future research will be committed to formulate further refinement steps to synthesize platforms from the optimal MEA architecture for closer implementation to an actual MEA EPDS.

\section{Appendix}

A generator's efficiency $\eta$ can be written as a relation between its mechanical input and its electrical output.

$$
\eta=\frac{\text { output }}{\text { input }}=\frac{\text { output }}{\text { output }+ \text { losses }}
$$

From the relationship above, the generator's losses can be found:

$$
\text { losses }=\text { output }\left(\frac{1-\eta}{\eta}\right)
$$

Let a generator $s$ have a power rating $P_{s}^{G}$, total supplied load of $\sum_{l} L_{l} y_{s l}$ (output), and a loading factor $\beta_{s}=\frac{\sum_{l} L_{l} y_{s l}}{P_{s}^{G}}$. The efficiency characteristic of this generator can be expressed either in terms of the power supplied (output) or its loading factor $\beta_{s}$ (see Fig. 1(b) in Section III). Considering an efficiency $\eta_{s}$ (in \%) in terms of the generator's loading factor $\beta_{s}$, the generator's losses $\eta_{s}^{\mathrm{LOSS}}$ (in $\mathrm{kW}$ ) can be written as:

$$
\eta_{s}^{\mathrm{LOSS}}=\left(\sum_{l} L_{l} y_{s l}\right)\left(\frac{100-\eta_{s}\left(\beta_{s}\right)}{\eta_{s}\left(\beta_{s}\right)}\right)
$$

Rewriting the expression above in terms of the loading factor $\beta_{s}$, the losses are: 


$$
\eta_{s}^{\mathrm{LOSS}}\left(P_{s}^{G}, \beta_{s}\right)=P_{s}^{G}\left(\frac{\sum_{l} L_{l} y_{s l}}{P_{s}^{G}}\right)\left(\frac{100-\eta_{s}\left(\beta_{s}\right)}{\eta_{s}\left(\beta_{s}\right)}\right)=P_{s}^{G} \beta_{s}\left(\frac{100-\eta_{s}\left(\beta_{s}\right)}{\eta_{s}\left(\beta_{s}\right)}\right)
$$

The losses can also be expressed in percentage (\%) of the power rating $P_{s}^{G}$,

$$
\eta_{s}^{\mathrm{LOSS}}\left(\beta_{s}\right)[\%]=100 \frac{\eta_{s}^{\mathrm{LOSS}}\left(P_{s}^{G}, \beta_{s}\right)}{P_{s}^{G}}=100 \frac{P_{s}^{G} \beta_{s}}{P_{s}^{G}}\left(\frac{100-\eta_{s}\left(\beta_{s}\right)}{\eta_{s}\left(\beta_{s}\right)}\right)=100 \beta_{s}\left(\frac{100}{\eta_{s}\left(\beta_{s}\right)}-1\right)
$$

\section{Acknowledgments}

This work is funded by the INNOVATIVE doctoral programme. The INNOVATIVE programme is partially funded by the Marie-Curie Initial Training Networks (ITN) action (project number 665468), and partially by the Institute for Aerospace Technology (IAT) at the University of Nottingham.

\section{References}

[1] A. S. Gohardani, G. Doulgeris, and R. Singh, "Challenges of future aircraft propulsion: A review of distributed propulsion technology and its potential application for the all electric commercial aircraft," Prog. Aerosp. Sci., vol. 47, no. 5, pp. 369-391, 2011.

[2] B. Sarlioglu and C. T. Morris, "More Electric Aircraft: Review, Challenges, and Opportunities for Commercial Transport Aircraft," IEEE Trans. Transp. Electrif., vol. 1, no. 1, pp. 54-64, 2015.

[3] G. Qiao, G. Liu, Z. Shi, Y. Wang, S. Ma, and T. C. Lim, “A review of electromechanical actuators for More/All Electric aircraft systems," Proc. Inst. Mech. Eng. Part C J. Mech. Eng. Sci., vol. 232, no. 22, pp. 4128-4151, 2018.

[4] G. Buticchi, S. Bozhko, M. Liserre, P. Wheeler, and K. Al-Haddad, "On-board microgrids for the more electric aircraft Technology review,” IEEE Trans. Ind. Electron., vol. 66, no. 7, pp. 5588-5599, 2019.

D. Hadbi, N. Retiere, F. Wurtz, X. Roboam, and B. Sareni, “Comparison between system design optimization strategies for more electric aircraft networks,” Int. J. Appl. Electromagn. Mech., vol. 53, no. S, pp. 289-305, 2015.

[6] Z. Duan et al., "An MILP method for design of distributed energy resource system considering stochastic energy supply and demand," Energies, vol. 11, no. 1, pp. 1-23, 2018.

[7] M. Maasoumy, P. Nuzzo, F. Iandola, M. Kamgarpour, A. Sangiovanni-Vincentelli, and C. J. Tomlin, "Optimal load management system for Aircraft Electric Power distribution,” in IEEE Conference on Decision and Control, 2013, pp. $2939-2945$.

[8] N. Bajaj, P. Nuzzo, M. Masin, and A. Sangiovanni-Vincentelli, "Optimized Selection of Reliable and Cost-Effective Cyber-Physical System Architectures," in Design, Automation \& Test in Europe Conference \& Exhibition (DATE), 2015, 
2015, pp. 561-566.

[9] S. Becz, A. Pinto, L. E. Zeidner, R. Khire, A. Banaszuk, and H. M. Reeve, "Design system for managing complexity in aerospace systems," in 10th AIAA ATIO/ISSMO Conference, 2010, pp. 1-7.

[10] P. Nuzzo et al., "A contract-based methodology for aircraft electric power system design,” IEEE Access, vol. 2, pp. 1-25, 2014.

[11] H. Kim, L. Guo, E. A. Lee, and A. Sangiovanni-Vincentelli, “A Tool Integration Approach for Architectural Exploration of Aircraft EPS with Ptolemy II / Metro II," in IEEE 1st International Conference on Cyber-Physical Systems, Networks, and Applications CPSNA, 2013, no. 1, pp. 38-43.

[12] A. Campbell, “Architecting Aircraft Power Distribution Systems via Redundancy Allocation,” 2014.

[13] P. Nuzzo, J. Finn, M. Mozumdar, and A. Sangiovanni-vincentelli, "Platform-Based Design Methodology and Modeling for Aircraft Electric Power Systems," in Proceedings of Green Energy and Systems Conference, 2013, pp. 1-7.

[14] R. D. Telford, S. J. Galloway, and G. M. Burt, "Evaluating the reliability \& availability of more-electric aircraft power systems," in Proceedings of the Universities Power Engineering Conference, 2012.

[15] T. Kostakis, P. Norman, S. Fletcher, S. Galloway, and G. Burt, "Evaluation of Paralleled Generation Architectures for Civil Aircraft Applications," in SAE, 2015, pp. 1-8.

[16] J. Chen, C. Wang, and J. Chen, "Investigation on the selection of electric power system architecture for future more electric aircraft," IEEE Trans. Transp. Electrif., vol. 4, no. 2, pp. 563-576, 2018.

[17] J. Bals, G. Hofer, A. Pfeiffer, and C. Schallert, "Virtual Iron Bird-a multidisciplinary modelling and simulation platform for new aircraft system architectures,” Dtsch. Luft-und Raumfahrtkongress, no. June, pp. 1-9, 2005.

[18] T. Kurtoglu, P. Bunus, J. De Kleer, and R. Rai, "Simulation-Based Design of Aircraft Electrical Power Systems," in International Modelica Conference, 2011, pp. 92-106.

[19] Y. Xu, Z. Zhang, J. Li, and Y. Yan, “Architecture analysis and optimization of high voltage DC parallel electric power system for more electric aircraft," in AUS 2016 - 2016 IEEE/CSAA International Conference on Aircraft Utility Systems, 2016, pp. 244-249.

[20] B. Wen et al., "Integrated Design by Optimization of Power Systems for More Electric Aircraft," in MEA 2015 - More Electric Aircraft -, 2015, no. February.

[21] X. Giraud et al., "Load allocation problem for optimal design of aircraft electrical power system," Int. J. Appl. Electromagn. Mech., vol. 43, no. 1-2, pp. 37-49, 2013.

[22] J. B. Hu, F. Li, J. Wu, J. H. Wang, and L. P. Zhang, "Research on Aviation Electric Power Cyber Physical Systems," Adv. Mater. Res., vol. 846-847, pp. 126-133, Nov. 2013.

[23] H. Xu, U. Topcu, and R. M. Murray, "Specification and synthesis of reactive protocols for aircraft electric power 
distribution," IEEE Trans. Control Netw. Syst., vol. 2, no. 2, pp. 193-203, 2015.

[24] H. Xu, N. Ozay, and R. M. Murray, “A Domain-Specific Language for Synthesis of Control Protocols for Aircraft Electric Power Systems," in Hybrid Systems: Computation and Control (HSCC) 2013, 2013, pp. 1-6.

[25] A. Sangiovanni-Vincentelli and G. Martin, "Platform-based design and software design methodology for embedded systems," IEEE Des. Test Comput., vol. 18, no. 6, pp. 23-33, 2001.

[26] X. Roboam et al., Integrated Design by Optimization of Electrical Energy Systems, 1st ed. UK: John Wiley \& Sons ISTE, 2012.

[27] H. Xu, U. Topcu, and R. M. Murray, “A case study on reactive protocols for aircraft electric power distribution,” 2012 IEEE 51st IEEE Conf. Decis. Control, pp. 1124-1129, 2012.

[28] T. Wongpiromsarn, U. Topcu, and R. M. Murray, "Receding horizon temporal logic planning," IEEE Trans. Automat. Contr., vol. 57, no. 11, pp. 2817-2830, 2012.

[29] T. Wongpiromsarn, U. Topcu, N. Ozay, H. Xu, and R. M. Murray, "TuLiP: A Software Toolbox for Receding Horizon Temporal Logic Planning," in Proceedings of the 14th international conference on Hybrid systems: computation and control, 2011, pp. 313-314.

[30] I. Filippidis, S. Dathathri, S. C. Livingston, N. Ozay, and R. M. Murray, "Control design for hybrid systems with TuLiP: The Temporal Logic Planning toolbox," in 2016 IEEE Conference on Control Applications, CCA 2016, 2016, pp. 10301041.

[31] A. Pinto, S. Becz, and H. Reeve, "Correct-by-Construction Design of Aircraft Electric Power Systems," in 10th AIAA Aviation Technology, Integration, and Operations (ATIO) Conference, 2010, pp. 1-11.

[32] C. Hang, P. Manolios, and V. Papavasileiou, "Synthesizing cyber-physical architectural models with real-time constraints," Lect. Notes Comput. Sci. (including Subser. Lect. Notes Artif. Intell. Lect. Notes Bioinformatics), vol. 6806 LNCS, pp. 441-456, 2011.

[33] A. Sangiovanni-Vincentelli, "Quo vadis, SLD? Reasoning about the trends and challenges of system level design," Proc. IEEE, vol. 95, no. 3, pp. 467-506, 2007.

[34] P. Wheeler and S. Bozhko, "The more electric aircraft: Technology and challenges," IEEE Electrification Magazine, vol. 2, no. 4, pp. 6-12, Dec-2014.

[35] A. Abdel-Hafez, "Power Generation and Distribution System for a More Electric Aircraft - A Review," Recent Adv. Aircr. Technol., 2012.

[36] I. Chakraborty, D. N. Mavris, M. Emeneth, and A. Schneegans, “An integrated approach to vehicle and subsystem sizing and analysis for novel subsystem architectures," Proc. Inst. Mech. Eng. Part G J. Aerosp. Eng., vol. 230, no. 3, pp. 496514, 2016. 
[37] S. Liscouët-Hanke, J.-C. Maré, and S. Pufe, "Simulation Framework for Aircraft Power System Architecting," J. Aircr., vol. 46, no. 4, pp. 1375-1380, 2009.

[38] A. Tardy et al., "Towards More Optimization for Aircraft Energy Conversion Systems," in MEA 2015 - More Electric Aircraft-, 2015, no. February, p. 16.

[39] H. Ounis, B. Sareni, X. Roboam, and A. De Andrade, "Multi-level integrated optimal design for power systems of more electric aircraft," in Mathematics and Computers in Simulation, 2016, vol. 130, pp. 223-235.

[40] E. Silvas, T. Hofman, N. Murgovski, L. F. P. Etman, and M. Steinbuch, "Review of Optimization Strategies for SystemLevel Design in Hybrid Electric Vehicles," IEEE Trans. Veh. Technol., vol. 66, no. 1, pp. 57-70, 2017.

[41] A. Recalde, S. Bozhko, J. Atkin, and C. Hill, "A reliability approach for the MEA power system architecture design optimization problem," in 2018 IEEE International Conference on Electrical Systems for Aircraft, Railway, Ship Propulsion and Road Vehicles \& International Transportation Electrification Conference (ESARS-ITEC), 2018, pp. 1-6.

[42] M. Minoux, "Networks synthesis and optimum network design problems: Models, solution methods and applications," Networks, vol. 19, no. 3, pp. 313-360, May 1989.

[43] B. Gendron, T. G. Crainic, and A. Frangioni, "Multicommodity Capacitated Network Design," in Telecommunications Network Planning, B. Sanso and P. Soriano, Eds. Boston, MA: Springer US, 1999, pp. 1-19.

[44] A. M. Costa, "A survey on benders decomposition applied to fixed-charge network design problems," Comput. Oper. Res., vol. 32, no. 6, pp. 1429-1450, Jun. 2005.

[45] Yu Liu and D. Tipper, "Successive survivable routing for node failures," in GLOBECOM 01 IEEE Global Telecommunications Conference, 2002, vol. 4, pp. 2093-2097.

[46] M. Garg and J. C. Smith, "Models and algorithms for the design of survivable multicommodity flow networks with general failure scenarios," Omega, vol. 36, no. 6, pp. 1057-1071, 2008.

[47] R. L. Y. Chen and C. A. Phillips, "K-Edge failure resilient network design,” Electron. Notes Discret. Math., vol. 41, pp. 375-382, 2013.

[48] A. K. Andreas and J. C. Smith, "Mathematical Programming Algorithms for Two-Path Routing Problems with Reliability Considerations," INFORMS J. Comput., vol. 20, no. 4, pp. 553-564, Nov. 2008.

[49] R. Sasaki, M. Wada, and O. Sakamoto, "Weight and Characteristics of Aircraft AC Generators Abstract," J. Japan Soc. Aeronaut. Sp. Sci., vol. 27, no. 305, pp. 296-300, 1979.

[50] a. Sangiovanni-Vincentelli, L. Carloni, F. De Bernardinis, and M. Sgroi, "Benefits and challenges for platform-based design," in Proceedings. 41st Design Automation Conference, 2004., 2004, pp. 409-414.

[51] X. Zhao, J. M. Guerrero, and X. Wu, "Review of aircraft electric power systems and architectures," in 2014 IEEE International Energy Conference (ENERGYCON), 2014, pp. 949-953. 
[52] L. Tarisciotti, A. Costabeber, C. Linglin, A. Walker, and M. Galea, "Evaluation of isolated DC/DC converter topolog for future HVDC aerospace microgrids,” 2017 IEEE Energy Convers. Congr. Expo. ECCE 2017, vol. 2017-Janua, pp. 2238$2245,2017$.

[53] T. Tholey, "Linear time algorithms for two disjoint paths problems on directed acyclic graphs," Theor. Comput. Sci., vol. 465, pp. 35-48, Dec. 2012.

[54] J. Brombach, A. Lucken, B. Nya, M. Johannsen, and D. Schulz, "Comparison of Different Electrical HVDC- Architectures for Aircraft Application," in Electrical Systems for Aircraft, Railway and Ship Propulsion (ESARS), 2012, 2012, pp. 1-6.

[55] N. A. of Sciences Engineering and Medicine and C. on Propulsion, Commercial Aircraft Propulsion and Energy Systems Research. Washington, D.C.: National Academies Press, 2016.

[56] “IBM ILOG CPLEX Optimization Studio.”[Online]. Available: https://www.ibm.com/products/ilog-cplex-optimizationstudio.

[57] S. Messaoud, “Optimal Architecture Synthesis for Aircraft Electrical Power Systems,” Technische Universitat Munchen, 2015 . 